\title{
Asteroseismic inference on the spin-orbit misalignment and stellar parameters of HAT-P-7 ${ }^{\star}$
}

\author{
Mikkel N. Lund ${ }^{1}$, Mia Lundkvist ${ }^{1,2}$, Victor Silva Aguirre ${ }^{1}$, Günter Houdek ${ }^{1}$, Luca Casagrande ${ }^{3}$, Vincent Van Eylen ${ }^{1}$, \\ Tiago L. Campante ${ }^{4,1}$, Christoffer Karoff ${ }^{5,1}$, Hans Kjeldsen ${ }^{1}$, Simon Albrecht ${ }^{1}$, William J. Chaplin ${ }^{4,1}$, \\ Martin Bo Nielsen ${ }^{6,7}$, Pieter Degroote ${ }^{8}$, Guy R. Davies ${ }^{4,1}$, and Rasmus Handberg ${ }^{4,1}$
}

\author{
${ }^{1}$ Stellar Astrophysics Centre, Department of Physics and Astronomy, Aarhus University, Ny Munkegade 120, 8000 Aarhus C, \\ Denmark \\ e-mail: mikkelnl@phys.au.dk \\ 2 Sydney Institute for Astronomy (SIfA), School of Physics, University of Sydney, NSW 2006, Australia \\ ${ }^{3}$ Research School of Astronomy \& Astrophysics, Mount Stromlo Observatory, The Australian National University, ACT 2611, \\ Australia \\ 4 School of Physics and Astronomy, University of Birmingham, Edgbaston, Birmingham, B15 2TT, UK \\ 5 Department of Geoscience, Aarhus University, Høegh-Guldbergs Gade 2, 8000, Aarhus C, Denmark \\ 6 Institut für Astrophysik, Georg-August-Universität Göttingen, Friedrich-Hund-Platz 1, 37077 Göttingen, Germany \\ 7 Max-Planck-Institut für Sonnensystemforschung, Justus-von-Liebig-Weg 3, 37077 Göttingen, Germany \\ 8 Instituut voor Sterrenkunde, Katholieke Universiteit Leuven, Celestijnenlaan 200D, 3001 Leuven, Belgium
}

Received 2 June 2014 / Accepted 28 July 2014

\begin{abstract}
Context. The measurement of obliquities - the angle between the orbital and stellar rotation - in star-planet systems is of great importance for understanding planet system formation and evolution. The bright and well-studied HAT-P-7 (Kepler-2) system is intriguing because several Rossiter-McLaughlin (RM) measurements found a high projected obliquity in this system, but it was not possible so far to determine whether the orbit is polar and/or retrograde.

Aims. The goal of this study is to measure the stellar inclination and hereby the full 3D obliquity of the HAT-P-7 system instead of only the 2D projection as measured by the RM effect. In addition, we provide an updated set of stellar parameters for the star. Methods. We used the full set of available observations from Kepler spanning Q0-Q17 to produce the power spectrum of HAT-P-7. We extracted oscillation-mode frequencies via an Markov chain Monte Carlo peak-bagging routine and used the results from this to estimate the stellar inclination angle. Combining this with the projected obliquity from RM and the inclination of the orbital plane allowed us to determine the stellar obliquity. Furthermore, we used asteroseismology to model the star from the extracted frequencies using two different approaches to the modelling, for which either the stellar evolution codes MESA or GARSTEC were adopted. Results. Our updated asteroseismic modelling shows, i.a., the following stellar parameters for HAT-P-7: $M_{\star}=1.51_{-0.05}^{+0.04} M_{\odot}, R_{\star}=$ $2.00_{-0.02}^{+0.01} R_{\odot}$, and age $=2.07_{-0.23}^{+0.28}$ Gyr. The modelling offers a high precision on the stellar parameters, the uncertainty on age, for instance, is of the order $\sim 11 \%$. For the stellar inclination we estimate $i_{\star}<36.5^{\circ}$, which translates into an obliquity of $83^{\circ}<\psi<111^{\circ}$. The planet HAT-P-7b is likely retrograde in its orbit, and the orbit is close to being polar. The new parameters for the star give an updated planetary density of $\rho_{\mathrm{p}}=0.65 \pm 0.03 \mathrm{~g} \mathrm{~cm}^{-3}$, which is lower than previous estimates.
\end{abstract}

Key words. asteroseismology - planetary systems - stars: oscillations - stars: individual: HAT-P-7 - methods: data analysis stars: rotation

\section{Introduction}

Asteroseismology can provide detailed information about stellar parameters such as mass, radius, and age (see Chaplin \& Miglio 2013, and references therein). Furthermore, an estimate for the stellar inclination can be obtained for solar-like oscillators (Gizon \& Solanki 2003), which in turn is needed in asserting the obliquity of planet-hosting systems. The obliquity of planetary systems, $\psi$, which is the angle between the stellar spin-axis and the angular momentum vector of the planetary orbit, is an important parameter for a better understanding of how these systems form and evolve (see, e.g., Nagasawa et al. 2008; Winn et al. 2010; Triaud et al. 2010; Morton \& Johnson 2011; Rogers et al. 2012). The obliquity is especially interesting

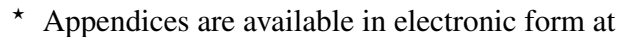
http: //www . aanda . org for systems that appear to have retrograde orbits from measurements of the sky-projected obliquity, $\lambda$. The reason is that while the orbit might indeed be retrograde, it is not known whether it is closer to polar than equatorial. This distinction makes a great difference for theories dealing with planetary system formation and evolution because they must be able to account for such a configuration (see, e.g., Chatterjee et al. 2008; Ford \& Rasio 2008; Matsumura et al. 2010; Lai et al. 2011; Albrecht et al. 2012).

Stellar obliquities are difficult to measure because stars are unresolved by modern telescopes. Therefore no spacial information can be obtained ${ }^{1}$. During planetary transits, however, parts of the stellar surface are covered, which breaks the degeneracy. Such information on the obliquity can be obtained, for instance, from studies of the anomalous effect in the radial velocity (RV) curve that is known as the Rossiter-McLaughlin (RM) effect

1 With the exception of stars with spacial interferometric constraints. 
(Rossiter 1924; McLaughlin 1924; see Fabrycky \& Winn 2009 for an overview). Unfortunately, only $\lambda$ can be obtained from RM measurements (see Fig. 1). Other means of obtaining $\lambda$ are spot-crossing anomalies that are observed during planetary transits (see, e.g., Sanchis-Ojeda et al. 2011, 2013; Désert et al. 2011), Doppler tomography (see, e.g., Gandolfi et al. 2012), or the effects of gravity darkening (see, e.g., Barnes et al. 2011; Ahlers et al. 2014), among others.

The true obliquity can only be unequivocally determined if the stellar angle of inclination, $i_{\star}{ }^{2}$, can be measured, and combined with $\lambda$ and the inclination of the planetary orbital plane, $i_{\mathrm{p}}$. The orbital inclination can be estimated fairly easily from analysis of the photometric light curve if the planet happens to transit its host star.

A measure of the stellar inclination angle can be obtained from combining $v \sin i_{\star}$ from spectroscopy with the stellar rotation period from modulations of the light curve from stellar spots (see Hirano et al. 2012, 2014, for recent uses of this method for planetary systems). These estimates can be quite uncertain because of the difficulty of calibrating the spectroscopic $v \sin i_{\star}$, separating the rotational signal from other broadening effects, and the need for an estimate of the stellar radius $R_{\star}$. A more direct method for obtaining $i_{\star}$ is that of asteroseismology, where the stellar inclination can be estimated by analysing solar-like acoustic ( $p$-mode) oscillations (Gizon \& Solanki 2003). Another great advantage of using asteroseismology is that a detailed stellar model can be obtained with well-determined parameters that are needed in simulations of planetary systems dynamics.

The high photometric quality that enabled the Kepler mission (see Borucki et al. 2010; Koch et al. 2010) to detect the transits of extrasolar planets, and thus allows for a determination of $i_{\mathrm{p}}$, also makes the data ideal for asteroseismic analysis (Gilliland et al. 2010). Analyses of the obliquity using the asteroseismic method have to date only been performed in the systems Kepler-50 and 65 by Chaplin et al. (2013), Kepler-56 by Huber et al. (2013a), Kepler-410 by Van Eylen et al. (2014), and 16 Cygni by Davies et al. (2014). However, for these systems the obliquity could only be assessed in a statistical sense, since the projected angle, $\lambda$, was unavailable.

Our aim in this paper is to use asteroseismology to determine precise stellar parameters of HAT-P-7 and to determine the obliquity of the HAT-P- $7^{3}$ system with a F6V (Faedi et al. 2013) type star and a close-in $\sim 1.78 M_{\text {J }}$ planet (HAT-P-7b) in a $\sim 2.2$ day orbit (Pál et al. 2008). From the very onset it is clear, however, that this is a challenging task because HAT-P-7 is a late-F-type star. This spectral type is notorious for having short lifetimes of the $p$-mode oscillations and consequently very broad (in frequency) oscillation modes, which highly obscures the potentially small effects imposed by rotation.

A fortuitous feature of the system is that not only $i_{\star}$ and $i_{\mathrm{p}}$ can be estimated from Kepler data, but the RM effect has been studied independently by Winn et al. (2009), Narita et al. (2009), and Albrecht et al. (2012) using HIRES and/or HDS data ${ }^{4}$. Values for $\lambda$ and $v \sin i_{\star}$ from these studies are given in Table 3 . Despite disagreement on the actual values, all three works agree

\footnotetext{
2 We define $i_{\star}$ as the angle between the stellar spin axis and the observer's line of sight, thus going from $i_{\star}=0^{\circ}$ for a pole-on view to $i_{\star}=90^{\circ}$ for an equator-on view.

3 We would like to emphasize the efforts made by O. Benomar and his collaborators for their work on HAT-P-7. This system was studied simultaneously and independently by our respective teams.

4 Keck-I/HIRES: High Resolution Spectrograph (Vogt et al. 1994); Subaru/HDS: High Dispersion Spectrograph (Noguchi et al. 2002).
}
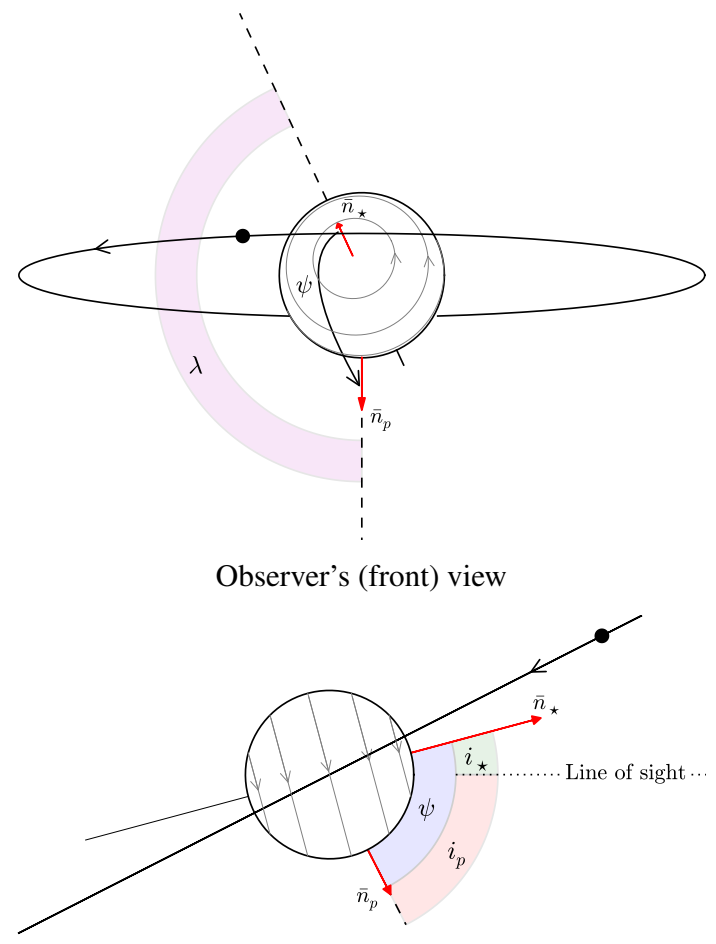

Side view

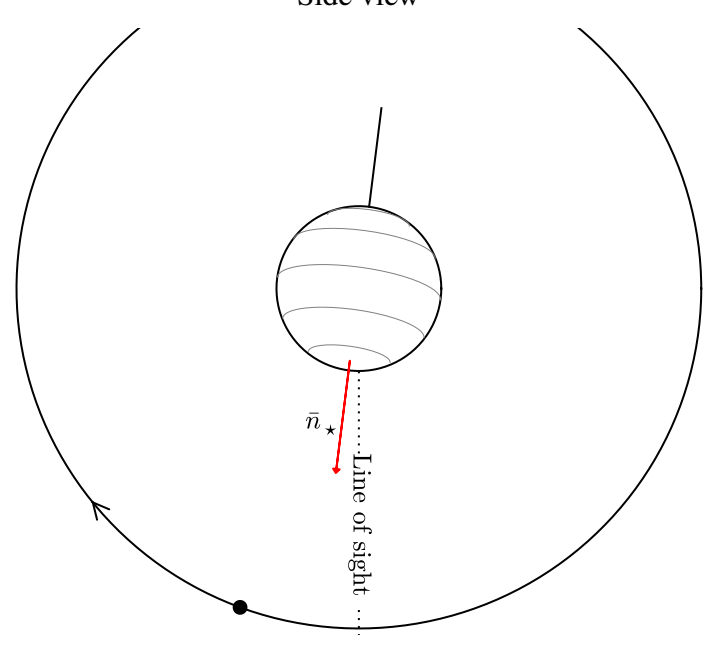

Top view

Fig. 1. Configuration of the HAT-P-7 system to scale using values for HAT-P-7b (full black circle) from Van Eylen et al. (2013). Top: the observer's view of the system from Earth, with the angular momentum vectors of the planetary orbit, $\bar{n}_{\mathrm{p}}$ (normal to the orbital plane), and stellar spin, $\bar{n}_{\star}$, given as red arrows. The projected angle, $\lambda$, is indicated by the shaded magenta region and is found as the angle between $\bar{n}_{\mathrm{p}}$ and $\bar{n}_{\star}$ when these are projected onto the plane of the sky (dashed lines). This angle is obtained from RM measurements, and in this panel we have used $\lambda=155 \pm 37^{\circ}$ following Albrecht et al. (2012; the uncertainty on $\lambda$ is not included in the figure). The stellar inclination, $i_{\star}$, which is the parameter measured from asteroseismology, is set to $15^{\circ}$, and is given by the direct angle between the line of sight (midpoint of star) and $\bar{n}_{\star}$. The inclination of the planetary orbit, $i_{\mathrm{p}}$, is set to $83^{\circ}$ following Van Eylen et al. (2013). The true angle, $\psi$, is the direct angle between $\bar{n}_{\mathrm{p}}$ and $\bar{n}_{\star}$. Middle: side view of the system, with the observer's view-point from the right, indicated by "line of sight". To properly show $i_{\star}$ (shaded green) and $i_{\mathrm{p}}$ (shaded red), and not their projected values, we have set $\lambda=180^{\circ}$ such that both $\bar{n}_{\mathrm{p}}$ and $\bar{n}_{\star}$ lie on the same plane as the line of sight. For illustrative purposes we have in addition decreased $i_{\mathrm{p}}$ to $63^{\circ}$. When adopting this configuration $\psi$ (shaded blue) is given by the sum of $i_{\star}$ and $i_{\mathrm{p}}$. Bottom: top view of the system, with the observer's view-point from the bottom, indicated by "line of sight". 
that the system is misaligned, that the planetary orbit might be retrograde, and that the very low value measured for $v \sin i_{\star} \operatorname{sug}$ gests a low $i_{\star}$, which for a transiting planet implies a near-polar orbit of the planet.

An interesting aspect of the system with regard to the obliquity is that a third body (an M5.5V dwarf known as HAT-P-7B) is found to be associated with the system (Narita et al. 2010, 2012; Bergfors et al. 2013; Faedi et al. 2013), and that a fourth associated body, likely more massive than Jupiter, is thought to be likely based on an unexplained RV excess (Winn et al. 2009; Narita et al. 2012). The system is thus a prime candidate for obliquity studies and theories concerned with planetary system formation and evolution.

The paper is structured as follows: in Sect. 2 we describe the data used in our analysis. Section 3 explains the model we used to obtain the stellar inclination angle. In Sect. 4 we present our analysis and results, including stellar modelling using two different codes (Sect. 4.2), and the results obtained for the stellar inclination and rotation (Sect. 4.3). Our results for the obliquity are the topic of Sect. 5. In Sect. 6 we compare our result on the stellar rotation rate with gyrochronology, and Sect. 7 is concerned with possible activity signatures. Finally, we discuss our results in Sect. 8 and conclude in Sect. 9.

\section{Data}

We extracted short-cadence $(\mathrm{SC} ; \Delta t=58.8 \mathrm{~s})$ simple aperture photometry (SAP) data from target pixel files (TPFs) using the procedure of Steven Bloemen (private communication), which starts from the original Kepler mask and adds to or removes pixels from the aperture based on the amount of signal in each pixel. The outcome of this is a new mask that most often is slightly larger than the original one.

The data span quarters from Q0 to Q17 ( 1470 days), with a duty cycle of $\sim 90.4 \%$. This constitutes the full amount of data available from Kepler in its normal mode of operation. Data were downloaded from the Mikulski Archive for Space Telescopes (MAST) and corrected using the procedure described in Handberg \& Lund (2014). Briefly, two median-filtered versions of the time series are computed with different filter windows. A weighted combination of the two are then used together with a filtered version of the planetary phase-curve to correct the time series for both instrumental features and the planetary signal. For the asteroseismic analysis we used filter windows of 1 ( $\left.\tau_{\text {long }}\right)$ and $0.028\left(\tau_{\text {short }}\right)$ days, while windows of $15\left(\tau_{\text {long }}\right)$ and $5\left(\tau_{\text {short }}\right)$ days were adopted in preparing the time series used in Sect. 7.2 to search for a low-frequency imprint of rotational modulation. We refer to Handberg \& Lund (2014) for further details on the filter and for a view of the corrected time series for HAT-P-7.

For the transit parameters needed in both the correction of the time series (orbital period) and for the estimation of the obliquity (inclination of orbital plane) we used the results of Van Eylen et al. (2013). The power spectrum was calculated using a weighted sine-wave fitting method (see, e.g., Kjeldsen 1992; Frandsen et al. 1995), normalised according to the amplitude-scaled version of Parseval's theorem (see Kjeldsen \& Frandsen 1992), in which a sine wave of peak amplitude, A, will have a corresponding peak in the power spectrum of $\mathrm{A}^{2}$.

\section{Fitting the power spectrum}

The harmonic eigenmodes of acoustic solar-like oscillations are characterised by their degree, $l$, which gives the number of nodal lines on the stellar surface, and their radial order, $n$, giving the number of radial nodes. In addition, an eigenmode is characterised by its azimuthal order, $m$, of which there are $2 l+1$. Only in the case of broken spherical symmetry, broken, for instance, by rotation, will the degeneracy between different $m$-values be lifted. The removal of this degeneracy makes it possible to measure the stellar rotation rate and inclination angle.

\subsection{Modelling the power spectrum}

We modelled the power spectral density of the oscillations with a series of standard Lorentzian functions. The limit spectrum (noise free) to be fit to the power spectrum can be expressed as follows:

$\mathcal{P}\left(v_{j} ; \boldsymbol{\Theta}\right)=\sum_{n=n_{a}}^{n_{b}} \sum_{l=0}^{2} \sum_{m=-l}^{l} \frac{\mathcal{E}_{l m}\left(i_{\star}\right) S_{n l}}{1+\frac{4}{\Gamma_{n l}^{2}}\left(v_{j}-v_{n l m}\right)^{2}}+B\left(v_{j}\right)$

Here $n_{a}$ and $n_{b}$ represent the first and last mode orders included from the power spectrum, while $v_{n l m}$ is the mode frequency including the effect of rotation, $B\left(v_{j}\right)$ describes the contribution from the stellar noise background at frequency $v_{j}, S_{n l}$ is the overall height of the multiplet, that is, the maximum power spectral density, and $\Gamma_{n l}$ is the mode line width. A geometrical modulation of the relative visibility between components of a split multiplet is given by $\mathcal{E}_{l m}\left(i_{\star}\right)$. The fitted parameters are denoted by $\boldsymbol{\Theta}$. This fit can be seen in the left panel of Fig. 2 .

The background signal $B(v)$ is described by a series of power laws (Harvey 1985), each of which relate to a specific physical phenomenon. The power laws included in this work describe the signals from granulation and faculae. The specific functional from for the background signal is that suggested by Karoff (2008):

$B(v)=\sum_{i=1}^{2} \frac{4 \sigma_{i}^{2} \tau_{i}}{1+\left(2 \pi v \tau_{i}\right)^{2}+\left(2 \pi v \tau_{i}\right)^{4}}+B_{0}$.

In this equation, $\sigma_{i}$ gives the flux rms variation in time and $\tau_{i}$ the characteristic time scales of the different phenomena. The constant $B_{0}$ is a measure of the photon shot-noise. The fit to the background can be seen in the right panel of Fig. 2 .

\subsection{Optimisation procedure}

The fit of Eq. (1) to the power spectrum is optimised in a Bayesian manner using the Markov chain Monte Carlo (MCMC) sampler emcee (Foreman-Mackey et al. 2013).

Frequencies for all modes are free parameters in the fit. Heights and widths are only free parameters for radial $(l=0)$ modes. For a non-radial mode the width is found from a linear interpolation between the two nearest radial modes. The same is true for the heights where the linearly interpolated value for the nearest radial modes is scaled via the visibility parameter (assumed constant as a function of frequency). In this work we keep the relative visibilities as free parameters. With this setup we have the following set of free parameters in the fitting: $\boldsymbol{\Theta}=\left\{v_{n l}, i_{\star}, v_{\mathrm{s}}, S_{n, 0}, \tilde{V}_{l=1}^{2}, \tilde{V}_{l=2}^{2}, \Gamma_{n, 0}\right\}$.

We refer to Appendix A for details on the fitted model and the adopted optimisation. 

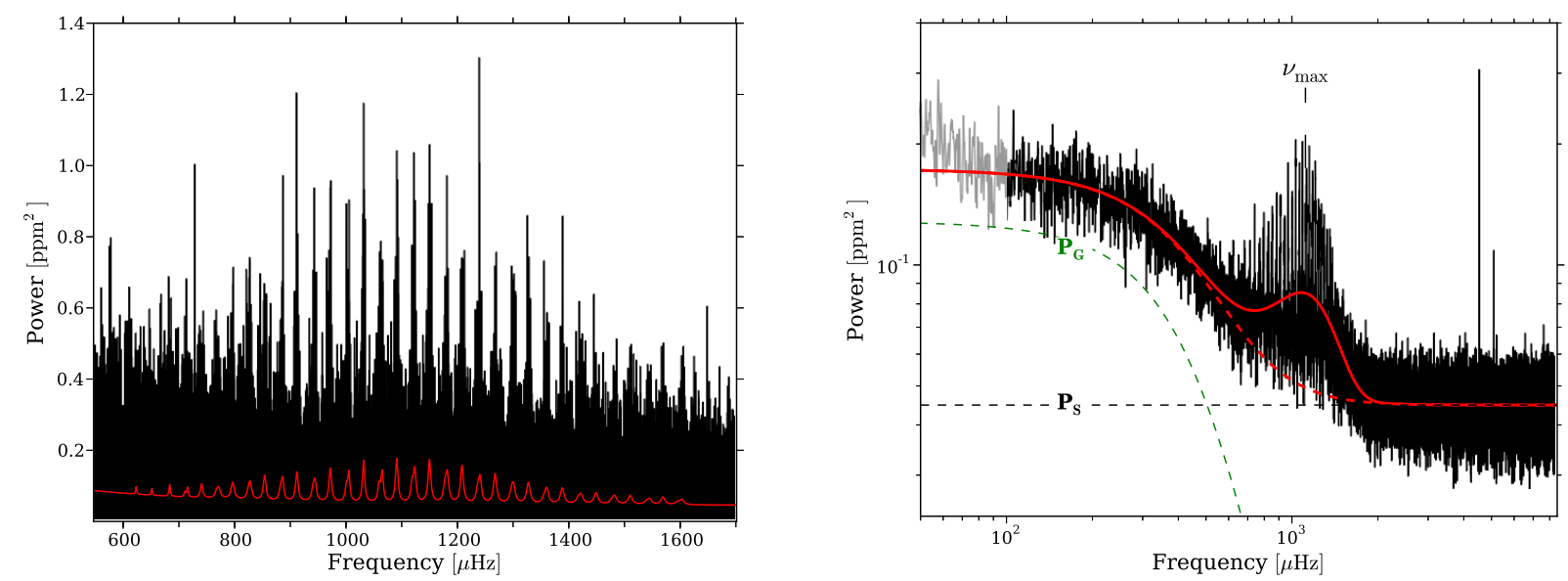

Fig. 2. Left: power spectrum of HAT-P-7 (black) with the fitted model over-plotted (red; cf. Eq. (1)). Right: power spectrum of HAT-P-7 (black) over-plotted with the best fit to the background (red; cf. Eq. (2)). The light-grey part up to $100 \mu \mathrm{Hz}$ was not included in the fit. The fit includes a granulation component $\left(\mathbf{P}_{\mathrm{G}}\right.$; green) and a white/shot noise $\left(\mathbf{P}_{\mathrm{S}}\right.$; black) level in addition to the Gaussian envelope from $p$-modes centred around $v_{\max } \approx 1115 \mu \mathrm{Hz}$. The dashed red line shows the background fit without the Gaussian envelope.

\section{Analysis and results}

\subsection{General results from peak-bagging}

To estimate mode frequencies for the modelling we fitted Eq. (1) to the frequency range $600-1650 \mu \mathrm{Hz}$, which is the range where we can visually identify modes. All estimated frequencies are given in Table D.1. Here we report the median of the marginalised posterior (kernel) probability distributions (PPDs) for the respective modes, while the uncertainties were obtained from the $68 \%$ highest probability density (HPD) credible region. For the frequency uncertainties used in the stellar modelling (see Sect. 4.2) we adopted the mean value of the (potentially asymmetric) uncertainties from the HPD credible region. For results on mode line widths and visibilities we refer to Appendix B.

To estimate the inclination and splitting parameters we fitted Eq. (1) to a smaller range in the power spectrum that included only the frequencies in the range $780-1400 \mu \mathrm{Hz}$. The selection of this interval was based on the estimates for the mode line widths from the large fit and to obtain modes with a relatively high signal-to-noise ratio $(\mathrm{S} / \mathrm{N})$. For the splitting we used a flat prior from -8 to $8 \mu \mathrm{Hz}$ and then used the absolute value of the splitting in Eq. (1). For the inclination we used a flat prior from -90 to $180^{\circ}$, and then folded values onto the interval from 0 to $90^{\circ}$. The symmetry of the priors was chosen to avoid potential boundary effects in the MCMC sampling.

We note that a component of Eq. (1) that might cause problems in the fitting is the noise-background. Initially, we did not fix the background in the fit of Eq. (1), but instead set Gaussian priors on the background parameters from the posteriors of a background-only fit. However, given that the background is very poorly constrained in the relatively small part of the power spectrum occupied by the oscillation modes, we found that not even the Gaussian priors were able to constrain the background, and especially the granulation time scale, $\tau_{\mathrm{g}}$, wandered to lower values. These lower values for $\tau_{\mathrm{g}}$ were found to correlate with a wide range of high values for the splitting at a particular value for the inclination. Because of this apparent degeneracy we chose to fix the background in the fits.

\subsection{Modelling of HAT-P-7}

A detailed modelling of HAT-P-7 was first made by Christensen-Dalsgaard et al. (2010) based on asteroseismic measurements of the solar-like $p$-mode oscillations in the star. This work was based on the SC Q0-Q1 data from the Kepler satellite. A fit of 33 individual mode frequencies, with values obtained from peaks of a smoothed power spectrum, was made to models computed using the Aarhus stellar evolution code (ASTEC; Christensen-Dalsgaard 2008a), with adiabatic pulsation frequencies calculated using the Aarhus adiabatic oscillation package (ADIPLS; Christensen-Dalsgaard 2008b).

Here we used the updated set of 50 frequencies from our peak-bagging to model HAT-P-7 and two different codes and modelling schemes to asses the robustness of our results. We note that this approach does not safeguard against other potential sources of systematics that can arise, for instance, from input physics that are not covered by the two codes or from assumptions made on certain quantities in the modelling. The effect of such systematics will be studied elsewhere (Silva Aguirre et al., in prep.).

The results from the modelling can be found in Table 1 . The échelle diagram (Grec et al. 1983) of HAT-P-7 is given in Fig. 3 after correcting for the background, and overlaid are both peak-bagged and modelled frequencies. In the construction of the échelle diagram we plot on the ordinate the medium frequency of the respective $\Delta v$-length segments. Here $\Delta v$ denotes the so-called large separation, computed as the frequency difference between consecutive radial orders of a given degree. To obtain a better representation of the ridges for illustrative purposes a constant value was added to the frequencies before taking the modulo, thus allowing a shift of the ridges on the abscissa (see, e.g., Bedding 2011). The $\Delta v$ from Huber et al. (2013b) was used.

\subsubsection{MESA model}

As a first approach, HAT-P-7 was modelled using Modules for experiments in stellar astrophysics (MESA; Paxton et al. 2011, 2013) and ADIPLS (Christensen-Dalsgaard 2008b). For the modelling we used the value of the effective temperature, $T_{\text {eff }}=6350 \pm 126 \mathrm{~K}$, the heavy-element abundance, $[\mathrm{Fe} / \mathrm{H}]=$ $+0.26 \pm 0.15$ (both from Huber et al. 2014), and the frequencies given in Table D.1, except for the lowest $l=0$ mode, which consistently agreed only extremely poorly with the models and was subsequently excluded.

For the input physics we chose to neglect diffusion and settling following Christensen-Dalsgaard et al. (2010). We used 
M. N. Lund et al.: Asteroseismic inference on the spin-orbit misalignment and stellar parameters of HAT-P-7

Table 1. Selected properties of our best-fit models compared with the first asteroseismic modelling by Christensen-Dalsgaard et al. (2010, CD10).

\begin{tabular}{llll}
\hline \hline & \multicolumn{3}{c}{ This Work } \\
\cline { 2 - 3 } Parameter & GARSTEC (preferred) & MESA & CD10 \\
\hline$M_{\star}\left[M_{\odot}\right]$ & $1.51_{-0.05}^{+0.04}$ & $1.63 \pm 0.09$ & 1.52 \\
$R_{\star}\left[R_{\odot}\right]$ & $2.00_{-0.02}^{+0.01}$ & $2.04 \pm 0.04$ & 1.992 \\
$L_{\star}\left[L_{\odot}\right]$ & $5.91_{-0.33}^{+0.31}$ & $6.2 \pm 0.5$ & 5.81 \\
Age $[\mathrm{Gyr}]$ & $2.07_{-0.23}^{+0.28}$ & $1.9 \pm 0.4$ & 1.875 \\
$\log g\left[\mathrm{~cm} \mathrm{~s}^{-2}\right]$ & $4.01_{-0.01}^{+0.01}$ & $4.03 \pm 0.01$ & 4.021 \\
$T_{\text {eff }}[\mathrm{K}]$ & $6366_{-80}^{+78}$ & $6374 \pm 130$ & 6355 \\
{$[\mathrm{Fe} / \mathrm{H}][\mathrm{dex}]$} & $+0.28_{-0.11}^{+0.11}$ & $+0.36 \pm 0.07$ & \\
$Y_{\text {ini }}$ & $0.288_{-0.008}^{+0.009}$ & $0.27 \pm 0.03$ & 0.2901 \\
$X_{\text {ini }}$ & $0.685_{-0.015}^{+0.013}$ & $0.70 \pm 0.03$ & 0.6809 \\
$M_{\text {core }}\left[M_{\star}\right]^{b}$ & $0.077_{-0.008}^{+0.007}$ & 0.076 & \\
$\alpha$ & 1.791 (fixed) & $1.88 \pm 0.16$ & 2.00 (fixed) \\
$f_{\text {ov }}$ & 0.016 (fixed) & $0.003 \pm 0.002$ & \\
\hline
\end{tabular}

Notes. The GARSTEC model (Sect. 4.2.2) constitutes our preferred values. ${ }^{(a)}$ Values from the model with the smallest $\chi^{2}$, with convective overshoot over 0.1 pressure scale heights included (model No. 2 in their Table 2). No uncertainties are reported by the authors. ${ }^{(b)}$ Mass of the convective core from the position of the Schwarzschild boundary. The overshooting region extends beyond this point.

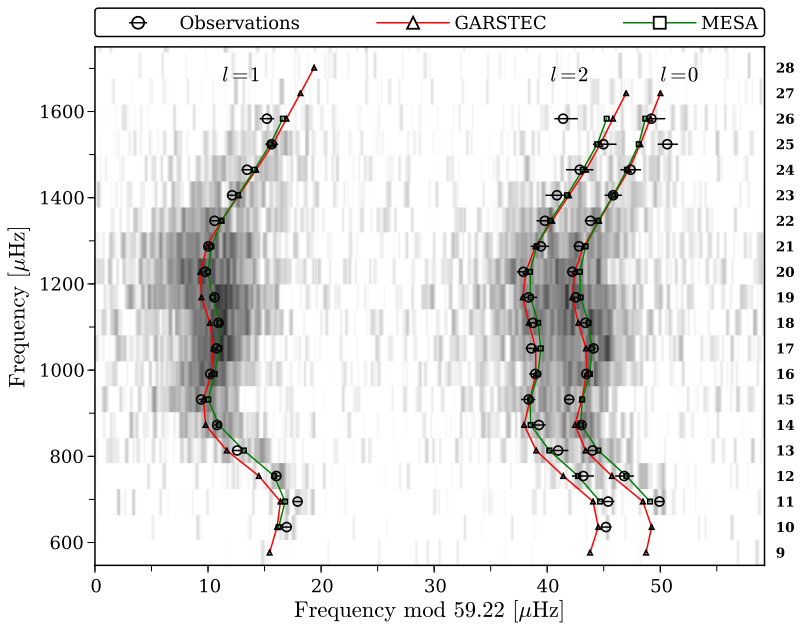

Fig. 3. Échelle diagram for HAT-P-7 using $\Delta v=59.22 \mu \mathrm{Hz}$. The grey scale ranges from white at low power to black at high power. Circles give the extracted frequencies with corresponding uncertainties, triangles connected by red lines give model frequencies from GARSTEC, while squares connected by green lines give model frequencies from MESA. The degree of each ridge is indicated in the top part of the plot. The radial order of the $l=0$ modes is indicated by the numbers at the right side of the plot.

the 2005 update of the OPAL EOS (Rogers et al. 1996; Rogers \& Nayfonov 2002) and the NACRE nuclear reaction rates (Angulo et al. 1999) with the updated ${ }^{14} \mathrm{~N}(p, \gamma){ }^{15} \mathrm{O}$ reaction rate by Formicola et al. (2004) and the updated ${ }^{12} \mathrm{C}(\alpha, \gamma){ }^{16} \mathrm{O}$ reaction rate by Kunz et al. (2002). Furthermore, we used the OPAL opacities (Iglesias \& Rogers 1996) assuming the solar chemical composition given by Asplund et al. (2009), supplemented by the Ferguson et al. (2005) opacities at low temperatures. We used the mixing-length theory of convection as formulated by Böhm-Vitense (1958). Finally, we chose to use the "simple photosphere" option in MESA for the atmospheric boundary condition, which constitutes a grey atmosphere with the optical depth, $\tau_{\mathrm{s}}$, to the base of the atmosphere of $2 / 3$ (see Paxton et al. 2011, their Eq. (3)).
In matching the model frequencies to the observed frequencies, we corrected the model frequencies for near-surface effects (Kjeldsen et al. 2008) using the prescription by Brandão et al. (2011) with $b=4.90$ and a reference frequency of $v_{0}=1100 \mu \mathrm{Hz}$. The best-fitting model was found via a $\chi^{2}$-minimisation (see, e.g., Christensen-Dalsgaard et al. 2010; Brandão et al. 2011; Doğan et al. 2013). The total $\chi^{2}$ was found as the weighted average of a normalised frequency and a spectroscopic component with weights given as $\chi^{2}=\frac{2}{3} \chi_{v}^{2}+\frac{1}{3} \chi_{\text {spectro }}^{2}$ (following Paxton et al. 2013) and the normalisation given by the number of values entering the $\chi^{2}$ component. The component $\chi_{\text {spectro }}$ included the match of model and spectroscopic values for $T_{\text {eff }}$ and $[\mathrm{Fe} / \mathrm{H}]$. The uncertainty of a given model parameter was found as the likelihood-weighted standard deviation of total $\chi^{2}$ values for all the computed models. Since we have chosen to use the model parameters for the best-fitting model instead of the likelihood-weighted mean values, we added the difference between the mean and the best-fitting values in quadrature to the uncertainties. The parameters of the best-fitting model and their uncertainties are listed in Table 1.

\subsubsection{GARSTEC model}

In the second approach we used two grids of stellar models computed with the Garching stellar evolution code (GARSTEC; Weiss \& Schlattl 2008).

The input physics is similar to the description given for the MESA model, with the difference that we used the mixinglength theory of convection of Kippenhahn et al. (2013), and Grevesse \& Sauval (1998) solar abundances. The updated ${ }^{12} \mathrm{C}(\alpha, \gamma){ }^{16} \mathrm{O}$ reaction rate was not used. In one grid we included the effects of core-overshooting using an exponential decay of the convective velocities with an efficiency of $f_{\mathrm{ov}}=0.016$ (Magic et al. 2010).

The grids cover a mass range between 0.7 and $1.8 M_{\odot}$ in steps of $0.01 M_{\odot}$ and initial compositions of $-0.65<[\mathrm{Fe} / \mathrm{H}]<$ +0.50 in steps of 0.05 dex. These were determined using a galactic chemical evolution law of $\Delta Y / \Delta Z=1.4$ (see, e.g., Balser 2006) anchored to the Big Bang nucleosynthesis value 

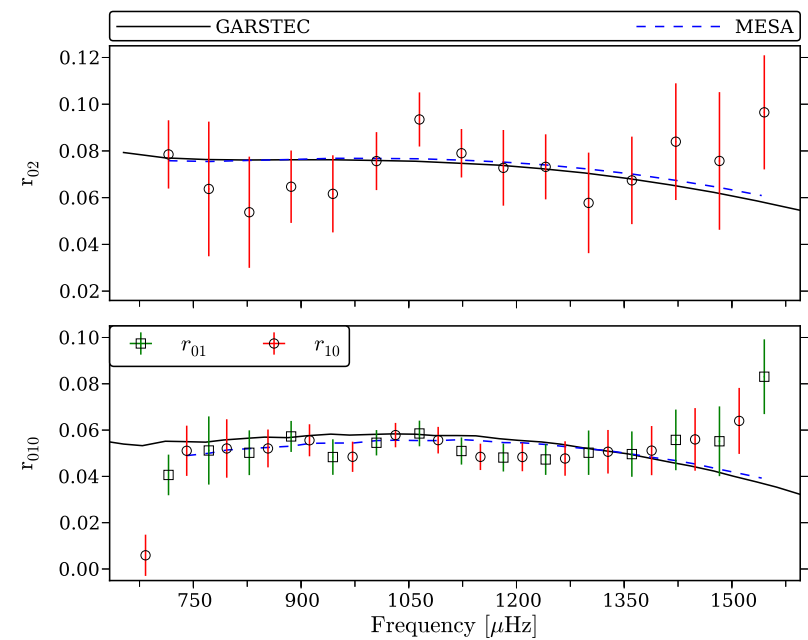

Fig. 4. Ratios $r_{02}$ and $r_{010}$ as a function of frequency. The lines show the ratios obtained for the best-fit GARSTEC (black solid) and MESA (dashed blue) models. Note that the relatively small uncertainties on frequencies (horizontal error bars) renders them indiscernible on this scale.

of $Y_{\mathrm{p}}=0.248$ (Steigman 2010). For hundreds of models along each evolutionary track, we computed theoretical oscillation frequencies using ADIPLS (Christensen-Dalsgaard 2008b). These allowed us to construct dense grids of models that covered the spectroscopic and asteroseismic parameter space of our target.

To determine the stellar parameters, we used the Bayesian approach described in Silva Aguirre et al. (in prep.). Briefly, we assumed a flat prior in $[\mathrm{Fe} / \mathrm{H}]$ and age including only a strict cut on the latter at $15 \mathrm{Gyr}$, and a standard Salpeter IMF (Salpeter 1955). We computed the likelihood of the observed values given a set of model parameters assuming Gaussian distributed errors. In our case, the observables included were the spectroscopic $T_{\text {eff }}$ and $[\mathrm{Fe} / \mathrm{H}]$, and the frequency ratios defined as (Roxburgh \& Vorontsov 2003)

$r_{01}(n)=\frac{d_{01}(n)}{\Delta v_{1}(n)}, \quad r_{10}(n)=\frac{d_{10}(n)}{\Delta v_{0}(n+1)}$

$r_{02}(n)=\frac{v_{n, 0}-v_{n-1,2}}{\Delta v_{1}(n)}$.

Here the $d_{01}$ and $d_{10}$ are the smooth five-point small frequency separations given as

$$
\begin{aligned}
& d_{01}(n)=\frac{1}{8}\left(v_{n-1,0}-4 v_{n-1,1}+6 v_{n, 0}-4 v_{n, 1}+v_{n+1,0}\right) \\
& d_{10}(n)=-\frac{1}{8}\left(v_{n-1,1}-4 v_{n, 0}+6 v_{n, 1}-4 v_{n+1,0}+v_{n+1,1}\right) .
\end{aligned}
$$

We refer to Silva Aguirre et al. (2013) for further details. In Fig. 4 we show the ratios obtained from the peak-bagging as a function of frequency along with ratios from the best-fit model. Here we also show the corresponding ratios obtained from the best-fit MESA model. Note that the ratios were not fitted in the MESA modelling. The construction of the ratios introduces correlations as a function of frequency that need to be taken into account when calculating the likelihood. This is done by calculating the $\chi^{2}$ entering the likelihood function as

$\chi^{2}=\frac{1}{N}\left(\boldsymbol{x}_{\text {obs }}-\boldsymbol{x}_{\text {model }}\right)^{T} \mathbf{C}^{-1}\left(\boldsymbol{x}_{\mathrm{obs}}-\boldsymbol{x}_{\text {model }}\right)$,

where $\mathbf{C}$ is the covariance matrix and the vectors $\boldsymbol{x}$ (of length $N$ ) give the observed and modelled values. As an illustration of $\mathbf{C}$ we show in Fig. 5 the Hinton diagram for the $r_{010}$ ratios, which

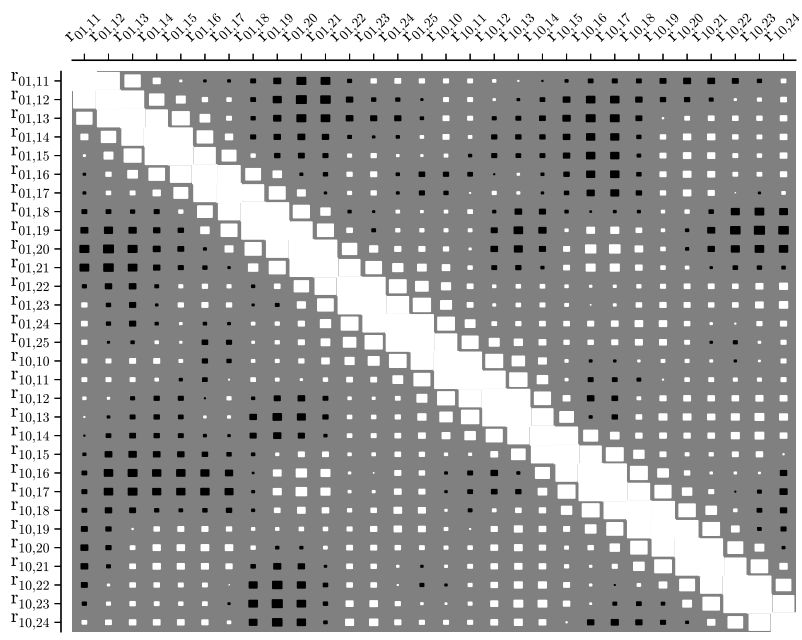

Fig. 5. Hinton diagram for the correlation matrix of $r_{010}$ ratios. The first part of the subscript denotes whether the ratio is of type 01 or 10 , while the second gives the radial order of the central frequency. White (black) squares indicate positive (negative) covariances between the ratios in question, and the size gives the relative size of the correlation (one along the diagonal).

provides a qualitative view of the correlation matrix for these ratios. To construct ratios we used the PPDs from the individual frequencies. From these distributions we obtained our central values and uncertainties and used the Pearson standard correlation coefficient ${ }^{5}$ to compute the correlation matrix.

We found that the grid that includes core-overshooting provided the best results. The final set of parameters from the modelling are given in Table 1. These were obtained from the median of the posterior probability distribution function and the 16 and 84 percent values. For the sake of comparison with the MESA model, the frequencies of the best-fit model are shown in Fig. 3 after applying the Kjeldsen et al. (2008) method to correct for near-surface effects. Note that the surface correction is not needed for this modelling approach because frequency ratios, which are not strongly affected by the surface layers (Roxburgh \& Vorontsov 2003; Silva Aguirre et al. 2011a), were used instead of the actual frequencies. Consequently, any possible apparent misfit between observed and surface corrected model frequencies in the échelle diagram cannot simply be interpreted as being caused by a poor model, but might just as well be due to a poor surface correction. In the current case, the fit, qualitatively speaking, reproduces the observations satisfactorily.

\subsubsection{Model comparison}

An improvement in our analysis compared with other works using Kepler data is that now the full available dataset can be utilised. Furthermore, the approach we adopted to extract mode frequencies, that is, using an MCMC peak-bagging scheme, is expected to provide more reliable estimates and uncertainties for the frequencies than the approach of assigning frequencies from peaks in a smoothed power spectrum.

In Table 1 we compare our results with the original asteroseismic results from Christensen-Dalsgaard et al. (2010). It is noteworthy that the GARSTEC model agrees quite well with these values, where only data from Q0-Q1 were used. The F-type character of the star limits the frequency precision by the large mode line widths (see Appendix B). This contributes to

5 Using the Python package Pandas. 
the relatively small changes in model results from the addition of significantly more data. Our MESA modelling results in a slightly more massive star than the stars obtained by GARSTEC and Christensen-Dalsgaard et al. (2010), but still agree within uncertainties despite the different fitting techniques.

The asteroseismic modelling by Van Eylen et al. (2012) resulted in a less massive $\left(M_{\star}=1.36 M_{\odot}\right)$ and less metal-rich $([\mathrm{Fe} / \mathrm{H}]=0.13)$ star than the current modelling efforts. This is likely because the authors explored a limited metallicity range and no spectroscopic constraints were included in the optimisation. Our stellar parameters agree with those derived by Pál et al. (2008) from a combined analysis of stellar isochrones, photometry, and spectroscopy. With our asteroseismic analysis we reduce the uncertainties on mass, radius, and age to $4.2 \%, 1.1 \%$, and $11.2 \%$, respectively, from the original estimates of $6.4 \%$, $13.9 \%$, and $45.5 \%$ given in Pál et al. (2008). In this comparison asymmetric uncertainties were added in quadrature.

Since the GARSTEC values are computed using frequency ratios that are insensitive to near-surface effects, this set of stellar parameters was adopted for the remainder of the paper. Furthermore, this Bayesian approach offers a direct estimation of the parameter uncertainties from the posterior distributions from a dense grid of models.

Finally, we note that the values of mass, radius, and age obtained from AME (Lundkvist et al. 2014); $M / M_{\odot}=1.55 \pm 0.05$, $R / R_{\odot}=1.99 \pm 0.02$, age $=1.9 \pm 0.5 \mathrm{Gyr}$, are consistent with the results from this work.

\subsubsection{Updated planetary parameters}

With our new estimates for the stellar parameters we can update the mass and radius for HAT-P-7b. The mass is found from (see, e.g., Winn 2010)

$$
\frac{M_{\mathrm{p}}}{\left(M_{\mathrm{p}}+M_{\star}\right)^{2 / 3}}=\frac{K_{\star} \sqrt{1-e^{2}}}{\sin i_{\mathrm{p}}}\left(\frac{P_{\text {orb }}}{2 \pi G}\right)^{1 / 3},
$$

where $M_{\mathrm{p}}$ is the planet mass, $e$ is the eccentricity, $P_{\text {orb }}$ is the orbital period, and $K_{\star}$ is the stellar reflex velocity. As input we use $i_{\mathrm{p}}$ and $P_{\text {orb }}$ from Van Eylen et al. (2013), $e$ is set to zero following Husnoo et al. (2012), and $K_{\star}=212.2 \pm 3.2 \mathrm{~m} \mathrm{~s}^{-1}$ from combining estimates of $K_{\star}$ by Winn et al. (2009) and Narita et al. (2009). Solving for the planet mass then gives ${ }^{6} M_{\mathrm{p}}=1.80 \pm$ $0.05 M_{J}$. For the planetary radius we derive $R_{\mathrm{p}}=1.51 \pm 0.02 R_{J}$ when using $R_{\mathrm{p}} / R_{\star}$ from Van Eylen et al. (2013). Considering the discussion in Van Eylen et al. (2013) on the size of systematic effects on transit depth measurements, we adopted a one per cent uncertainty on $R_{\mathrm{p}} / R_{\star}$. This gives a planetary density of $\rho_{p}=$ $0.65 \pm 0.03 \mathrm{~g} \mathrm{~cm}^{-3}$, which is just within the uncertainty of the estimate from Pál et al. (2008) of $\rho_{p}=0.876_{-0.24}^{+0.17} \mathrm{~g} \mathrm{~cm}^{-3}$. We note that the stellar parameters estimated from asteroseismology offers a greatly improved precision on the planetary parameters.

\subsection{Splitting and inclination}

In Fig. 6 we present the results for the splitting and inclination of HAT-P-7 as the PPDs for these parameters, along with the 2D correlation map. These results were obtained from the small fit described above. The $68 \%$ and $95 \%$ HPD credible regions are indicated in Fig. 6.

The distributions for inclination and splitting for HAT-P-7 are unfortunately not simple and Gaussian, but rather cover an

\footnotetext{
6 Using $M_{J}=1.899 \times 10^{30} \mathrm{~g}$ and $R_{J}=7.1492 \times 10^{9} \mathrm{~cm}$.
}

Table 2. Values related to the stellar inclination and rotation.

\begin{tabular}{lc}
\hline \hline Parameter & $68 \%$ HPD limit \\
\hline$i_{\star}\left[{ }^{\circ}\right]$ & $<36.5$ \\
$v_{\mathrm{s}}[\mu \mathrm{Hz}]$ & $<0.87$ \\
$P_{\text {rot }}[$ days $]$ & $>13.23$ \\
$v \sin i_{\star}\left[\mathrm{km} \mathrm{s}^{-1}\right]$ & $<2.21$ \\
$v_{\text {surf }}\left[\mathrm{km} \mathrm{s}^{-1}\right]$ & $<7.66$ \\
\hline
\end{tabular}

Notes. All values are estimated from the $68 \%$ HPD credible region of their corresponding parameter distributions.

extended region of parameter space. Therefore we refrain from using measures of central tendency, such as the median or mode of the distributions, but instead report values from the 68\% HPD credible regions. The overall behaviour seen in most of the correlation map corresponds well to what might be expected given the relatively large line widths: $\Gamma=4.8-8.4 \mu \mathrm{Hz}$ for the range fitted (see Fig. B.1). At low inclinations, the central $m=0$ component dominates the relative heights of azimuthal components of a rotationally split multiplet, while at high inclinations the sectoral $(m= \pm l)$ components dominate. For $l=2$ the tesseral $(0<|m|<l)$ components dominate at an inclination of $\sim 50^{\circ}$. Thereby, the splitting is generally less constrained at low inclinations because the $m \neq 0$ components are small and harder to fit. Furthermore, a line width exceeding the splitting effectively hides these small components up to a splitting of half the line width (i.e., up to $\sim 4 \mu \mathrm{Hz}$ ). At higher inclinations the splitting is more easily discernible because the visible azimuthal components are the ones farthest apart, thus the line width becomes less of a problem.

There is a high density at $i_{\star} \sim 10^{\circ}$ and $v_{\mathrm{s}}>5 \mu \mathrm{Hz}$. This should not be interpreted as a good solution to the fit of the power spectrum but is rather the result of walkers trying to escape the parameter space, with the consequence that the walkers pile up at the boundary of the prior on the splitting. A contributing factor could also be that the small separation between $l=0$ and $l=2$ modes is of the order $\sim 5 \mu \mathrm{Hz}$, whereby $l=2$ azimuthal components would be within the $l=0$ mode profile. We see no correlations between these high splitting values and any of the other parameters of the fit and so these parameters are unaffected by this feature. These walkers will, however, contribute to the shape of the splitting and inclination PPDs, and therefore we chose to exclude walkers with $v_{\mathrm{s}}>5 \mu \mathrm{Hz}$ in the marginalisation of the $i_{\star}$ and $v_{\mathrm{S}}$ PPDs. This is the same effect as observed when the background was kept free in the fit, but now much decreased. It is, however, not clear to us what about the inclination of $i_{\star} \sim 10^{\circ}$ facilitates this escape of walkers.

From the $68 \%$ HPD credible regions of the inclination and splitting PPDs we find $i_{\star}<36.5^{\circ}$ and $v_{\mathrm{s}}<0.87 \mu \mathrm{Hz}$ (see Table 2). The value for the inclination agrees overall with previous reports that were based on the low value for $v \sin i_{\star}$. In addition, agreement is found with the statistically derived estimate by Schlaufman (2010) of $i_{\star}=6.7-10^{\circ}$ based on a simple $P_{\text {rot }} \propto t^{1 / 2}$ model (Weber \& Davis 1967; Skumanich 1972) for the evolution of the stellar rotation period as a function of mass and age. The splitting, on the other hand, is lower than expected for an F6-type star (see below). However, it is clear from the correlation map that at low inclinations, values of the splitting up to $\sim 4 \mu \mathrm{Hz}$ are allowed. 

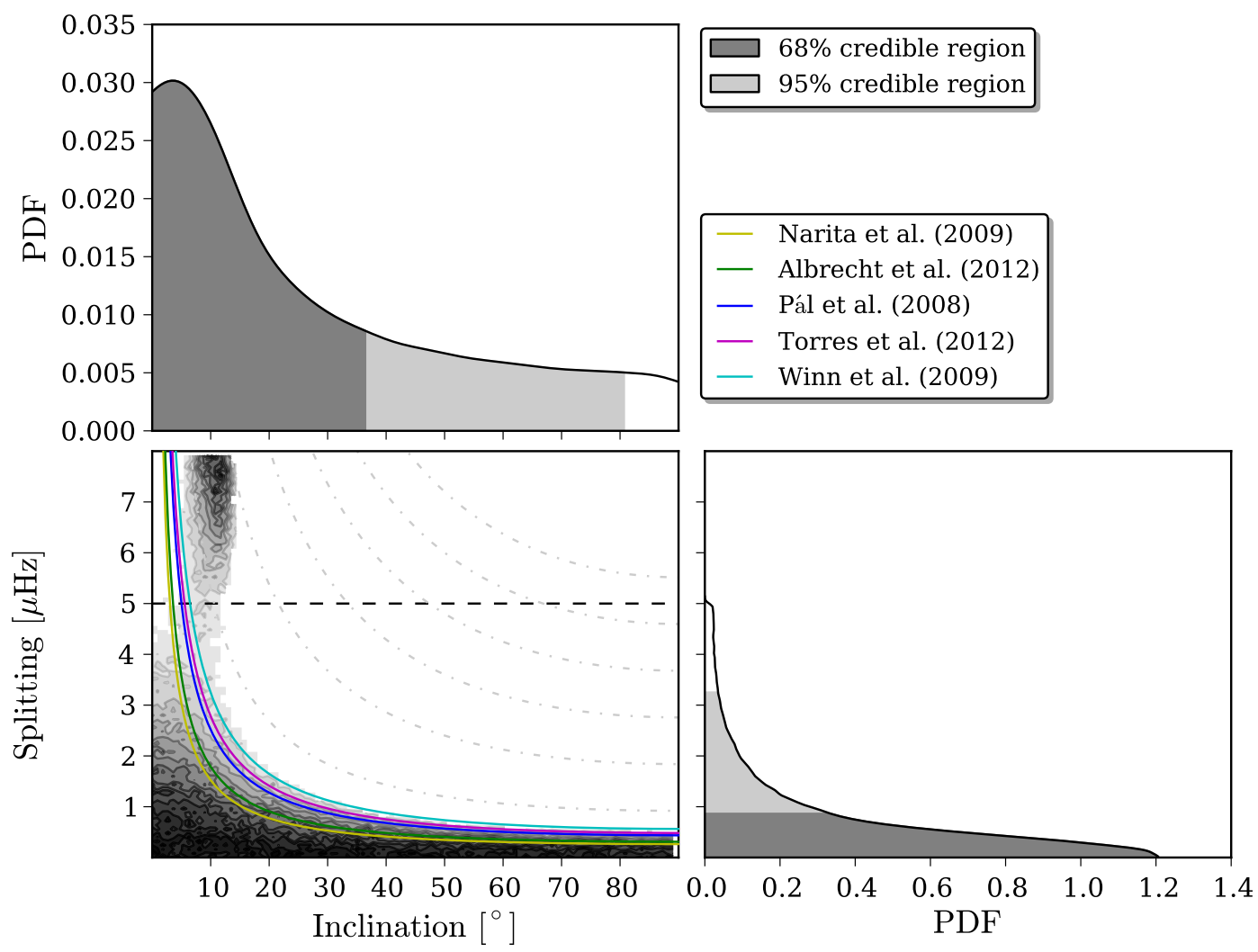

Fig. 6. Top: marginalised posterior probability distribution (PPD) for the stellar inclination $i_{\star}$. Here we have folded the full distribution $\left(-90\right.$ to $\left.180^{\circ}\right)$ onto the the range from 0 to $90^{\circ}$. Bottom right: PPD for the rotational splitting. Bottom left: correlation map between the inclination and the rotational splitting. The $68 \%$ credible regions (highest posterior density credible regions) are indicated by the dark-grey parts, while light-grey indicates the additional parts covered in a $95 \%$ credible region. Included are also lines of constant $v \sin i_{\star}$, computed using the radius estimate from our analysis and with $v \sin i_{\star}$ values from the literature (see Table 3 ), arranged in the legend in order of increasing $v \sin i_{\star}$ value (top to bottom). The dashed black horizontal line indicates the value of $v_{\mathrm{s}}=5 \mu \mathrm{Hz}$ above which walkers were excluded from the PPDs. The grey dash-dotted lines give lines of constant $v \sin i_{\star}$ from 8 to $48 \mathrm{~km} \mathrm{~s}^{-1}$ in steps of $8 \mathrm{~km} \mathrm{~s}^{-1}$.

Table 3. Literature values for parameters related to the HAT-P-7 system.

\begin{tabular}{lcccc}
\hline \hline Source & $v \sin i_{\star}\left[\mathrm{km} \mathrm{s}^{-1}\right]$ & $\lambda\left[^{\circ}\right]$ & $i_{\mathrm{p}}\left[^{\circ}\right]$ & $\psi$ (this work) \\
\hline Pál et al. (2008) & $3.8 \pm 0.5$ & & $85.7_{-3.1}^{+3.5}$ & \\
Winn et al. (2009) & $4.9_{-0.9}^{+1.2}$ & $182.5 \pm 9.4$ & $80.8_{-1.2}^{+2.8}$ & $83^{\circ}<\psi<119^{\circ}$ \\
Narita et al. (2009) & $2.3_{-0.5}^{+0.6}$ & $-132.6_{-16.3}^{+10.5 a}$ & $85.7_{-3.1}^{+3.5}$ & $83^{\circ}<\psi<106^{\circ b}$ \\
Albrecht et al. (2012) & $2.7 \pm 0.4$ & $155 \pm 37^{c}$ & & $83^{\circ}<\psi<111^{\circ}$ \\
Torres et al. (2012) & $4.2 \pm 0.5$ & & & \\
Van Eylen et al. (2013) & & $83.151_{-0.033}^{+0.030}$ & \\
This work & $<2.21$ & & & \\
\hline
\end{tabular}

Notes. ${ }^{(a)}$ The value for the projected obliquity is equivalent to $\lambda=227.4_{-16.3}^{+10.5 \circ} \cdot{ }^{(b)}$ We used an uncertainty on $\lambda$ of $\pm 16.3^{\circ} .{ }^{(c)}$ From a fit to RM data Albrecht et al. (2012) obtained an uncertainty on $\lambda$ of $\pm 14^{\circ}$. The uncertainty adopted here was ascribed by Albrecht et al. (2012) as the standard deviation of the three independent measurements of $\lambda$.

With our estimate for the stellar radius (see Sect. 4.2) we may convert the estimated ranges for the splitting and inclination to a measure of $v \sin i_{\star}$ as (Chaplin et al. 2013)

$v \sin i_{\star}=2 \pi R v_{\mathrm{s}} \sin i_{\star}$.

Using the PPDs obtained for $v_{\mathrm{s}}$ and $i_{\star}$, while assuming a distribution for the radius as $R / R_{\odot} \sim \mathcal{N}(2.00,0.02)$, we find value of $v \sin i_{\star}<2.21 \mathrm{~km} \mathrm{~s}^{-1}$ (68\% HPD credible region). In Fig. 7 we have plotted the full distribution for $v \sin i_{\star}$ and indicate the values obtained from spectral and RM analysis (see Table 3).
We find that the value of Narita et al. (2009) agrees within uncertainties with our results. Winn et al. (2009) found the highest value for $v \sin i_{\star}$. Their higher $v \sin i_{\star}$ value results from the lower orbital inclination value (see Table 3 ) they used to model the RM effect. For HAT-P-7 a larger impact parameter (lower inclination) requires a larger $v \sin i_{\star}$. If the analysis were repeated with the inclination derived from the Kepler light curve instead of the ground-based data that were available to them, this disagreement in $v \sin i_{\star}$ would vanish.

After measuring $v \sin i_{\star}$ and $i_{\star}$ and assuming solid-body rotation, we can now calculate the true rotation speed of HAT-P-7, 
M. N. Lund et al.: Asteroseismic inference on the spin-orbit misalignment and stellar parameters of HAT-P-7

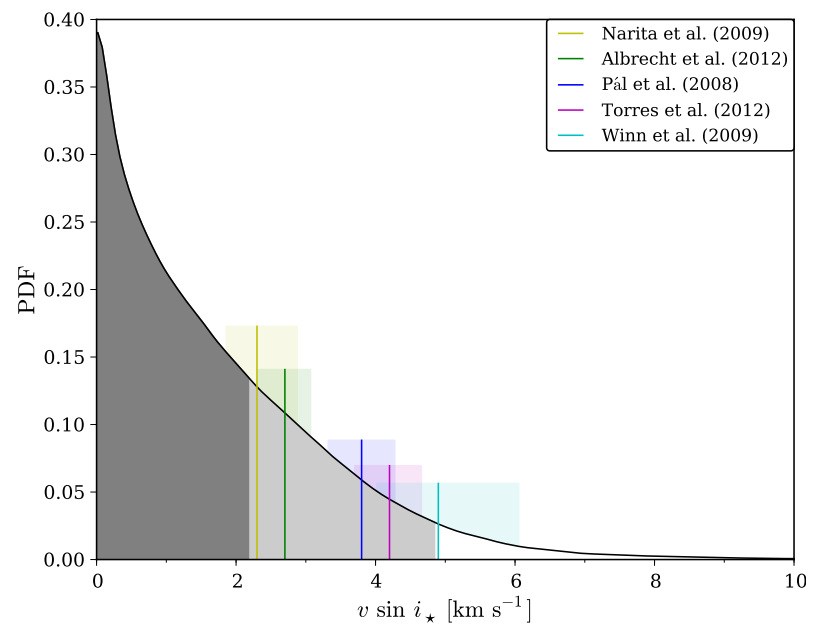

Fig. 7. Distribution for $v \sin i_{\star}$ constructed from the PPDs obtained for $v_{\mathrm{S}}$ and $i_{\star}$ and assuming $R / R_{\odot} \sim \mathcal{N}(2.00,0.02)$ (see Eq. (9)). The dark- and light-grey regions of the distribution correspond to the $68 \%$ and $95 \%$ credible regions. Literature values for $v \sin i_{\star}$ obtained from spectral and RM analysis (see Table 3 ) are given by vertical lines where the shaded regions above the distribution give the corresponding uncertainties.

$v<7.66 \mathrm{~km} \mathrm{~s}^{-1}$ (68\% HPD credible region). This upper-limit estimate agrees with the values from Nielsen et al. (2013) for the rotation periods of Kepler stars of an approximate spectral type between F4 and 6 .

\section{Obliquity}

With our estimate for the stellar inclination, $i_{\star}, \lambda$, and the planetary orbital inclination, $i_{\mathrm{p}}$, we are now able to calculate the system obliquity, $\psi$, from (Winn et al. 2005)

$\cos \psi=\sin i_{\star} \cos \lambda \sin i_{\mathrm{p}}+\cos i_{\star} \cos i_{\mathrm{p}}$.

In Fig. 8 we show the distribution for $\psi$ for the obtained distribution for $i_{\star}$ (see Fig. 6), while adopting $i_{\mathrm{p}}$ from Van Eylen et al. (2013), and $\lambda$ from Albrecht et al. (2012). From the 68\% HPD credible region we obtain $83^{\circ}<\psi<111^{\circ}$, consistent with a polar orbit. The corresponding results using $\lambda$ from Winn et al. (2009) and Narita et al. (2009) are given in Table 3.

We refer to Albrecht et al. (2012) for a discussion on the different values for $\lambda$ and the possible reasons for their disagreement (see also Albrecht et al. 2011). Here we note that regardless of which $\lambda$-value we used we found a polar orbit for HAT-P-7b.

When the obliquity is assessed without knowing the stellar inclination, a flat distribution in $\cos i_{\star}$ is generally assumed for the stellar orientation, because this results in an isotropic distribution for the stellar inclination. From this distribution it is a priori much more likely to observe a random star in an equator-on configuration. In Fig. 8 we show the distribution in $\psi$ from adopting this isotropic distribution in $i_{\star}$. In this way, Winn et al. (2009) estimated $\psi>86.3^{\circ}$ with $99.73 \%$ confidence, while Narita et al. (2009) found $\psi>90^{\circ}$ with $99.70 \%$ confidence (both used $i_{\mathrm{p}}$ from Pál et al. 2008). From this approach a retrograde orbit is thus strongly suggested, but the orbit has a higher probability of being more equatorial than polar. With our asteroseismic estimate for the inclination we can substantiate these statistical results because we find $\psi>90^{\circ}$ with $68 \%$ credibility using $\lambda$ from Albrecht et al. (2012), and now a near-polar orbit is the most likely configuration for the system.

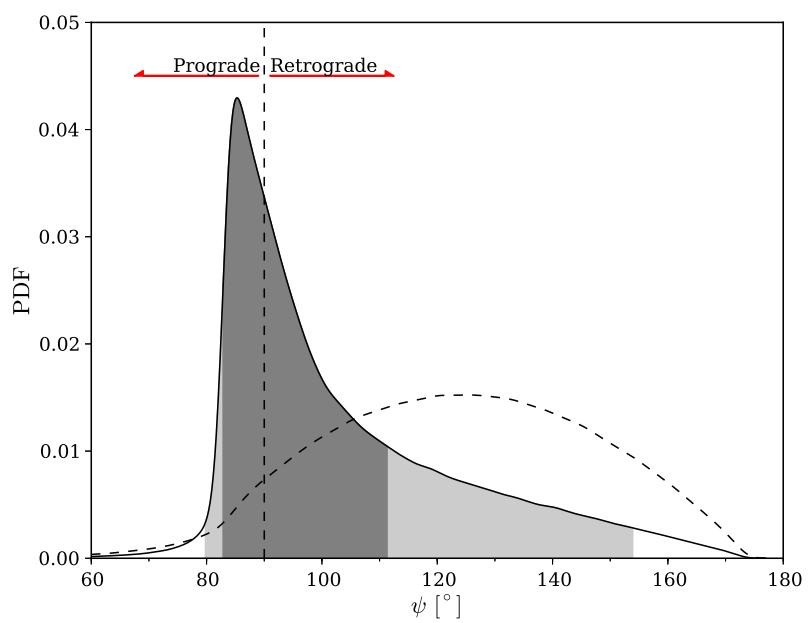

Fig. 8. Distribution of the true angle $\psi$ using the distribution for $i_{\star}$ from the peak-bagging, and with the assumption of normal distributions for the planetary inclination $i_{\mathrm{p}}$ and projected angle $\lambda$. For $i_{\mathrm{p}}$ the value from Van Eylen et al. (2013) was adopted, while $\lambda$ was taken from Albrecht et al. (2012). The dark- and light-grey regions of the distribution correspond to the $68 \%$ and $95 \%$ credible regions. The dashed curve gives the distribution assuming instead an isotropic distribution for $i_{\star}$, i.e., flat in $\cos i_{\star}$. We indicate also which values of $\psi$ correspond to a retrograde or prograde orbit of HAT-P-7b.

\section{Comparison with gyrochronology}

Our result for the limits on the stellar rotation rate (see Table 2) can be compared with empirically calibrated gyrochronology relations. Here we use the form described by Barnes (2007) given as

$P(B-V, t)=t^{n} \times a\left[(B-V)_{0}-c\right]^{b}$,

where $t$ is the stellar age in Myr, while $a, b$, and $n$ are empirically determined coefficients that vary depending on the calibration set used (see, e.g., Epstein \& Pinsonneault 2014). To compare with this relation, we first need an estimate for the de-reddened colour $(B-V)_{0}$. The procedure used to derive $(B-V)_{0}$ is described in Appendix $C$.

Using the estimate $(B-V)_{0}=0.495 \pm 0.022$ together with the age determined in Sect. 4.2 of $t=2.07 \pm 0.36$ Gyr (see Table 1; asymmetric uncertainties were added in quadrature), we can estimate the rotation period from the relation in Eq. (11). In Fig. 9 we show two versions of this relation, those by Barnes (2007) (B07), and those by Meibom et al. (2009; M09; see also Barnes $\&$ Kim 2010; Meibom et al. 2011). From these we compute periods of $9.9 \pm 1.8$ (B07) and $5.0 \pm 4.0$ days (M09). The upper value from the B07 relation is similar to our lower-limit period estimate of about 13 days (see Table 2), which means that the agreement is not very convincing. On the other hand, the splittings at low inclinations match this range of rotation periods well because the level of $\sim 4 \mu \mathrm{Hz}$ corresponds to a rotation period of $\sim 2.9$ days (see Fig. 6).

We note that a $P_{\text {rot }} \propto t^{1 / 2}$ law might provide a poor description of the rotational evolution for HAT-P-7, because its $(B-V)_{0}$ places it in close proximity to the so-called Kraft break (Kraft 1967) where loss of angular momentum via a stellar wind is inhibited by the lack of a sufficiently deep convection zone. If HAT-P-7 is on the low $(B-V)_{0}$ side of this break, the rotation rate becomes a strong function of the initial conditions (see, e.g., van Saders \& Pinsonneault 2013), and a gyrochronology scaling is not applicable. 


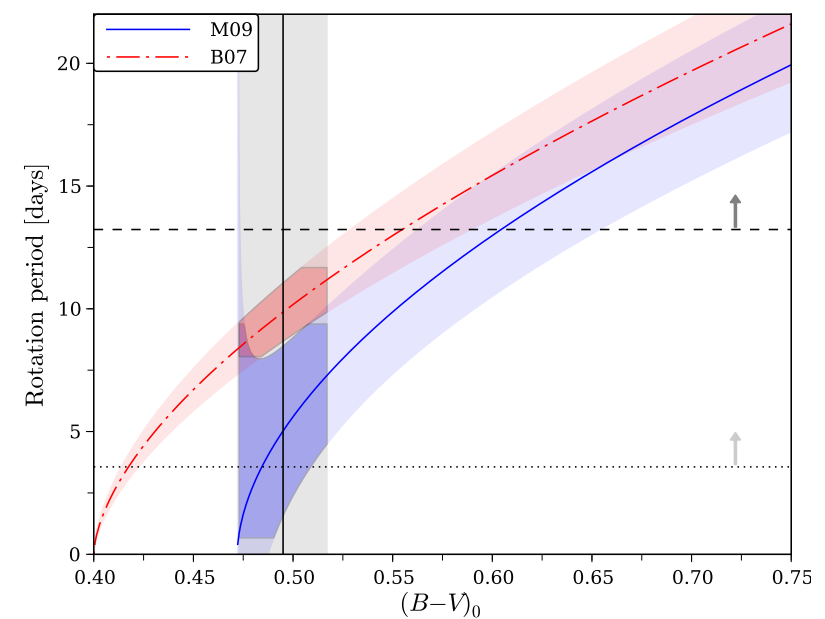

Fig. 9. Gyrochronology relations from Meibom et al. (2009; M09) and Barnes (2007; B07). The shaded region around each relation (with the same colour) represents the standard error of the relationship between $(B-V)_{0}$ and rotation period from propagating the uncertainties reported for the coefficients entering Eq. (11) together with the uncertainty in our age estimate. The vertical line and shaded region give the value of $(B-V)_{0}=0.495 \pm 0.022$ of HAT-P-7, while darker shaded regions around the relations vertically bound the corresponding uncertainty in the period from the uncertainty in $(B-V)_{0}$, age, and the gyrochronology relations. The horizontal lines indicate the limits on the $68 \%$ (dashed) and $95 \%$ (dotted) credible regions on the stellar rotation period from $p$-mode splittings. The upward-pointing arrows indicate that these rotation periods are lower limits from the respective HPD credible regions.

The close-in hot-Jupiter HAT-P-7b has potentially had an impact on the rotation rate of its host star. While a detailed dynamical analysis of this system is beyond the scope of this paper, the synchronisation of rotation and alignment clearly has not been reached yet. The time scale for circularisation has been reached, however, with an upper limit on the eccentricity of $e<0.038$ given by Husnoo et al. (2012). While it is difficult to assess the past interaction between the planet and the star, we note that with the configuration we estimate for the system, that is, close to a polar orbit of the planet, the rotational synchronisation time will likely be very long because the angular momentum vectors are close to being orthogonal, thereby decreasing the tidal interaction. For discussions on the interaction between hot Jupiters and their host stars we refer to Hut (1981), Miller et al. (2009), Cohen et al. (2010), Matsumura et al. (2010), Mardling (2011), and Valsecchi \& Rasio (2014), for example.

\section{Activity signatures}

Activity of stars is linked to the interaction between rotation, convection, and magnetic fields. The interaction can cause magnetic features, such as dark spots and bright faculae, to appear in the photosphere of the stars (see, e.g., Berdyugina 2005, for a review), and plages in the chromosphere. If a star is rotating, such dark or bright regions will induce a temporal modulation of the integrated stellar flux.

The photospheric features can also cause departures from radiative equilibrium in the stellar chromosphere in plages, and induce emission in the cores of specific spectral lines. For example, emission in cores of $\mathrm{Ca}$ II H\&K lines is an often used indicator of magnetic activity (Knaack et al. 2001; Wright et al. 2004; Frasca et al. 2011; Fröhlich et al. 2012) because it is believed to reflect the amount of non-thermal chromospheric heating above faculae (see, e.g., Hall 2008, for a review). For close-in
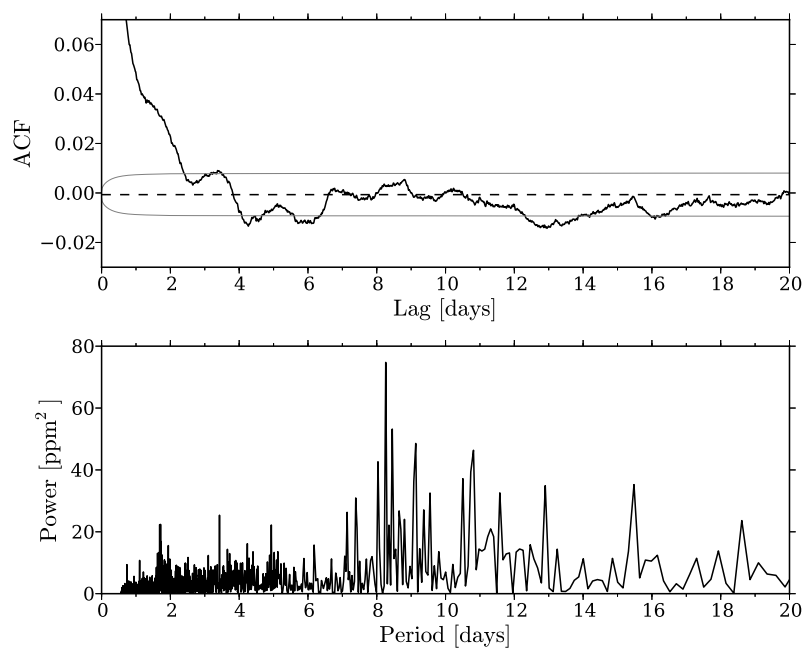

Fig. 10. Top: autocorrelation function (ACF) of the HAT-P-7 time series (black). The dashed horizontal line gives the expectation value for random independent and identically distributed values, while the grey curves give the large-lag 95\% confidence levels. Bottom: low-frequency end of the power spectrum in units of period.

hot-Jupiter systems there is furthermore the possibility for a direct magnetic interaction between the planet and the star, where magnetic reconnections, similar to those seen in flares, can cause a heating of the chromosphere (see, e.g., Shkolnik et al. 2005).

\subsection{Chromospheric activity}

To test for signatures of chromospheric activity we searched the Ca II H\&K lines in the HIRES spectra from Winn et al. (2009), but found no signs of emission or high levels of activity. Because we see $p$-mode oscillations in HAT-P-7, it must have an outer convection zone, and the $(B-V)_{0}$ colour derived in Sect. 6 places it above the limit from Simon \& Landsman (1991) for the onset of activity. The absence of an emission signal could point towards an intrinsically low surface activity caused by a low rotation rate.

Another effect that would influence the signal is if HAT-P-7 has a low angle of inclination. On the Sun, faculae are primarily located in the active latitude bands between around 5 and $40^{\circ}$ latitude. As faculae and plages are believed to have the same magnetic driver the $\mathrm{Ca}$ II $\mathrm{H} \& \mathrm{~K}$ emission from plages will thus decrease with decreasing inclination from the decreasing projected area of the active regions (Knaack et al. 2001; Freire Ferrero et al. 2004; Chaplin et al. 2007).

\subsection{Low-frequency region}

We searched for a signal imparted by a temporal flux modulation at low frequencies in the power spectrum of HAT-P-7 (see, e.g., Campante et al. 2011; Nielsen et al. 2013). Examples of such activity signals in F-type Kepler stars can be seen, for instance, in Mathur et al. (2014).

The signal from the stellar rotation can also be estimated for example by the autocorrelation function (ACF) of the time series, as shown for instance by McQuillan et al. (2013). In Fig. 10 we show the ACF of the corrected time series together with the low-frequency end of the power spectrum (in units of period for convenience). The ACF shows no clear sign of modulation. To test the hypothesis that a signal in the time series has died out at lag $k$, we used the large-lag standard error (Anderson 1976), 


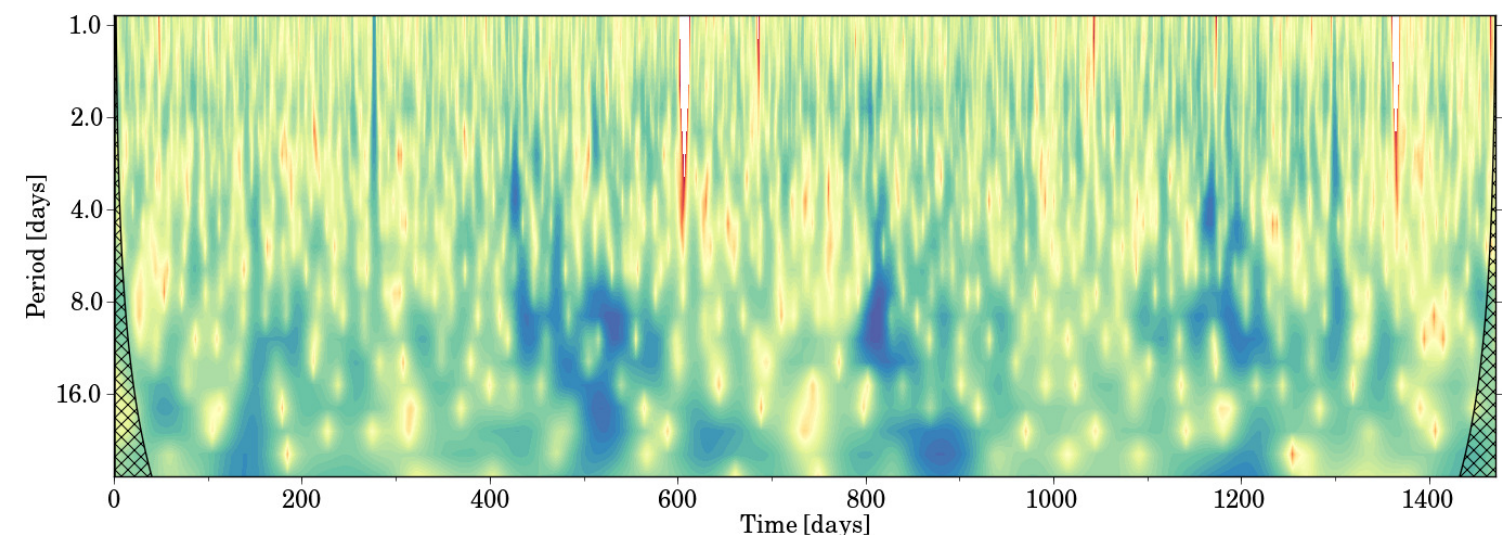

Fig. 11. Morlet wavelet power spectrum as a function of time for HAT-P-7, where the time series was binned by 31 points. The colour changes from red at low power to blue at high power - the colours are on a logarithmic scale. The cross-hatched regions at high and low times indicate the cone of influence where edge effects become important (see Torrence \& Compo 1998). No clear signatures from rotation are apparent.

indicated in Fig. 10. From this it is clear that the signal seen in the ACF is not significant. However, the low-amplitude hump at around $\sim 9$ days in the ACF does seem to align with an increase in power in the power spectrum.

We investigated the presence of a modulation also via the Morlet wavelet transform (Torrence \& Compo 1998) of the time series (see also Mathur et al. 2013), this is shown in Fig. 11. No clear periodicity is seen, and the signal that causes the $\sim 9$ day hump in the ACF and the power spectrum seems to be very intermittent. Finally, we checked the magnetic proxy from Campante et al. (2014), but we did not find indications of a signal here either. The fact that a stronger or more localised signal is missing for HAT-P-7 might again be linked to a low stellar inclination. Indeed, if magnetic features such as stellar spots primarily reside near the equator of the star, while we view it close to pole-on, a strong modulation in the light curve would not be expected.

We also note a collection of peaks in the power spectrum around a frequency of $6.7 \pm 0.4 \mu \mathrm{Hz}$ (corresponding to a period of $\sim 41.6 \mathrm{~h}$ ). The presence of this power excess is quite robust and is consistently seen in random segments of the total time series and in the wavelet spectrum. We therefore rule out that it originates from random noise. First, we checked that there are no known artefacts at this frequency (Christiansen et al. 2013). To determine whether the signal might originate from another star in the vicinity of HAT-P-7 $\left(K_{\mathrm{p}}=10.463\right)$ we located all Kepler targets with available data within a radius of $3^{\prime}$ (12 were found). Of these, the star KIC 10666727 $\left(K_{\mathrm{p}}=13.166\right)$ was found to have strong signatures of spot modulations in its time series and broadened power excess peaks in its power spectrum around $\sim 2.2 \mu \mathrm{Hz}, \sim 4.6 \mu \mathrm{Hz}$, and most interestingly, in the region $6.7-7.2 \mu \mathrm{Hz}$. The angular separation between this target and HAT-P-7 is around 155", which places the two stars close enough for direct PRF (pixel response function) contamination to occur between them (Coughlin et al. 2014). However, according to Coughlin et al. (2014), stars have to be bright to contaminate over such a large separation. We do not see any significant power excess in HAT-P-7 at other frequencies where KIC 10666727 shows an even stronger excess than at $6.7 \pm 0.4 \mu \mathrm{Hz}$. Therefore we find it possible, but unlikely, that KIC 10666727 is contaminating the light curve of HAT-P-7.

\section{Discussion}

With asteroseismic modelling we have provided a precise and detailed model for HAT-P-7, with parameters that can be used in tests of theories on the formation and evolution of planetary systems. These, together with the obliquity, are especially important to constrain when attempting to explain a system such as HAT-P-7, which likely has a close-to polar orbit of its hot-Jupiter planet.

Our estimate of the obliquity of the HAT-P-7 system supports the hypothesis described by Winn et al. (2010), who stated that hot Jupiters are born with a wide range of obliquities. Planet-planet scatterings (Chatterjee et al. 2008) and the effect of Kozai cycles and tidal friction (Fabrycky \& Tremaine 2007) could for example create large initial obliquities. For cool dwarfs with deep convection zones, tidal dissipation would operate efficiently and result in aligned systems. Hot stars fail to align because they lack such a deep convection zone for most of their life on the main-sequence, if not altogether. This hypothesis was put forth based on the observed trend of $\lambda$ against $T_{\text {eff }}$, where a broad distribution is seen in $\lambda$ for $T_{\text {eff }}>6250 \mathrm{~K}$, while predominantly low values for $\lambda$ are seen below this temperature. This result was corroborated by Albrecht et al. (2012) based on a larger sample of measurements. These authors also found that the systems with respect to $\lambda$ could be sorted into relative tidal time-scales, which in turn depend on the planet to star mass ratio, and $a / R_{\star}$ ( $a$ being the semimajor axis), among others. While HAT-P-7 falls on the hot side of the dividing temperature between the two regimes and tidal interactions are expected to be weak, it is worth noting that the temperature is quite close to this dividing line. Moreover, $a / R_{\star}$ is small, which increases tidal interaction (Albrecht et al. 2012). The relatively slow rotation we found suggests that some magnetic braking has taken place and is still ongoing. The lack of alignment is likely linked to the combined effects of a low mass of the convection zone (making tides ineffective), the close-to orthogonal alignment of the angular momentum vectors, and the relatively young age of the system. Indeed, the star might have started out with a more rapid rotation, where magnetic braking from the developing convection zone has been more effective in slowing down the star than has the tidal interaction in realigning the system.

With regard to the obliquity of the system, the fact that a third, and possibly a fourth, body is found to be associated (Winn et al. 2009; Narita et al. 2012) might support the scenario of fewbody dynamical interaction early in the life of the system, which resulted in the high obliquity of HAT-P-7b.

\section{Conclusions}

Using asteroseismology, we have estimated the stellar inclination and provided new and precise stellar parameters for 
HAT-P-7 based on the extracted mode frequencies. Mode frequencies were extracted using a Bayesian MCMC approach to peak-bag the frequency power spectrum from corrected Kepler data, for which we utilised the full SC dataset from Q0-Q17 available from the Kepler satellite. From this, information was obtained about stellar, planetary, and system parameters (age, mass, radius, composition, luminosity, mass of convective core) that are important ingredients, for instance, in dynamical simulations used to test theories for the evolution of planetary systems.

We found the star of the HAT-P-7 system to have a low inclination, $i_{\star}<36.5^{\circ}$ with $68 \%$ credibility, meaning that the star is seen close to pole-on. Combining this with estimates for the planetary orbital inclination and the projected obliquity from RM measurements, the close-in hot-Jupiter planet is in a high obliquity and likely retrograde orbit (the retrograde solutions account for $\sim 68 \%$ of the PDF in Fig. 8). Our estimate for the stellar rotation matches empirical findings for stars of the same spectral type, and using the age of the system from our modelling of the oscillation frequencies in combination with an improved estimate for the colour of the star (see Appendix C) yields that estimates from gyrochronology are not in conflict with our results. While the lack of signatures from activity are by no means proof of a low inclination for the star, they are not incompatible with a low inclination.

To our knowledge, this analysis is the first wherein asteroseismology has been able to provide an estimate for $i_{\star}$ that, together with $\lambda$ from RM measurements and $i_{\mathrm{p}}$ from analysis of the transit profile, has allowed for a near complete description of the system geometry.

For theories attempting to explain the formation and evolution of planetary systems, the HAT-P-7 system is highly interesting because any such theory must be able to offer an explanation for the system geometry. The analysis presented in this paper has shed more light on the obliquity of the system, and not just the projected obliquity that is normally used in obliquity studies. The result on the obliquity agrees with assumptions based on the high value of $\lambda$ and the low value for $v \sin i_{\star}$. Furthermore, the high value for $\psi$ corroborates the theory where the degree of alignment is connected to the tidal evolution of the system (see, e.g., Winn et al. 2010; Albrecht et al. 2012; Valsecchi \& Rasio 2014).

An aspect of the HAT-P-7 system that might be investigated to constrain the obliquity even better is the apparent asymmetry of the transit light curve, seen for instance in the phase curve presented in Esteves et al. (2013) and Van Eylen et al. (2013). Such an asymmetry is also seen in the KOI-13 system, for example, and it was found by Szabó et al. (2011) and Barnes et al. (2011) to agree well with the predictions by Barnes (2009) for a planet crossing over the gravity-brightened polar region of its rapidly rotating host star on a high-obliquity orbit.

Acknowledgements. The authors wish to thank the entire Kepler team, without whom these results would not be possible. We are thankful to O. Benomar and his collaborators for their approach to the contemporaneousness of our respective studies. We would like to thank Eric Stempels and Heidi Korhonen for useful discussions in the early phases of the project. Thanks to Joshua N. Winn and John A. Johnson for making their Keck spectra available to us. Funding for the Stellar Astrophysics Centre (SAC) is provided by The Danish National Research Foundation. The research is supported by the ASTERISK project (ASTERoseismic Investigations with SONG and Kepler) funded by the European Research Council (Grant agreement No. 267864). M.L. would like to thank the asteroseismology group at SIfA for their hospitality during a research stay where some of this work was carried out. M.L. also wishes to thank Niels Bohr Fondet for financial support for the research stay at SIfA. T.L.C., G.R.D., W.J.C., and R.H. acknowledge the support of the UK Science and Technology Facilities Council (STFC). C.K. acknowledges the support of the Villum Foundation. M.B.N. acknowledges research funding by Deutsche Forschungsgemeinschaft (DFG) under grant SFB 963/1 "Astrophysical flow instabilities and turbulence" (Project A18). The research leading to these results has received funding from the European Community's Seventh Framework Programme (FP7/2007-2013) under grant agreement No. 269194. This research has made use of the following web resources: the SIMBAD database (simbad.u-strasbg.fr), operated at CDS, Strasbourg, France; NASAs Astrophysics Data System Bibliographic Services (adswww.harvard.edu); arxiv.org, maintained and operated by the Cornell University Library.

\section{References}

Ahlers, J. P., Seubert, S. A., \& Barnes, J. W. 2014, ApJ, 786, 131 Albrecht, S., Winn, J. N., Johnson, J. A., et al. 2011, ApJ, 738, 50 Albrecht, S., Winn, J. N., Johnson, J. A., et al. 2012, ApJ, 757, 18 Anderson, E. R., Duvall, Jr., T. L., \& Jefferies, S. M. 1990, ApJ, 364, 699 Anderson, O. 1976, Time series analysis and forecasting: the Box-Jenkins approach (Butterworth)

Angulo, C., Arnould, M., Rayet, M., et al. 1999, Nucl. Phys. A, 656, 3

Appourchaux, T., Benomar, O., Gruberbauer, M., et al. 2012, A\&A, 537, A134 Asplund, M., Grevesse, N., Sauval, A. J., \& Scott, P. 2009, ARA\&A, 47, 481 Ballot, J., Barban, C., \& van't Veer-Menneret, C. 2011, A\&A, 531, A124

Balser, D. S. 2006, AJ, 132, 2326

Barnes, J. W. 2009, ApJ, 705, 683

Barnes, J. W., Linscott, E., \& Shporer, A. 2011, ApJS, 197, 10

Barnes, S. A. 2007, ApJ, 669, 1167

Barnes, S. A., \& Kim, Y.-C. 2010, ApJ, 721, 675

Bedding, T. R. 2011 [arXiv: 1107. 1723]

Benomar, O., Appourchaux, T., \& Baudin, F. 2009, A\&A, 506, 15

Berdyugina, S. V. 2005, Liv. Rev. Sol. Phys., 2

Bergfors, C., Brandner, W., Daemgen, S., et al. 2013, MNRAS, 428, 182

Böhm-Vitense, E. 1958, ZAp, 46, 108

Borucki, W. J., Koch, D., Basri, G., et al. 2010, Science, 327, 977

Brandão, I. M., Doğan, G., Christensen-Dalsgaard, J., et al. 2011, A\&A, 527, A37

Campante, T. L., Handberg, R., Mathur, S., et al. 2011, A\&A, 534, A6

Campante, T. L., Chaplin, W. J., Lund, M. N., et al. 2014, ApJ, 783, 123

Casagrande, L., \& VandenBerg, D. A. 2014, MNRAS, submitted [arXiv: 1407.6095]

Casagrande, L., Ramírez, I., Meléndez, J., Bessell, M., \& Asplund, M. 2010, A\&A, 512, A54

Casagrande, L., Ramírez, I., Meléndez, J., \& Asplund, M. 2012, ApJ, 761, 16

Casagrande, L., Silva Aguirre, V., Stello, D., et al. 2014, ApJ, 787, 110

Chaplin, W. J., \& Miglio, A. 2013, ARA\&A, 51, 353

Chaplin, W. J., Elsworth, Y., Houdek, G., \& New, R. 2007, MNRAS, 377, 17 Chaplin, W. J., Sanchis-Ojeda, R., Campante, T. L., et al. 2013, ApJ, 766, 101 Chatterjee, S., Ford, E. B., Matsumura, S., \& Rasio, F. A. 2008, ApJ, 686, 580 Christensen-Dalsgaard, J. 2008a, Ap\&SS, 316, 13

Christensen-Dalsgaard, J. 2008b, Ap\&SS, 316, 113

Christensen-Dalsgaard, J., Kjeldsen, H., Brown, T. M., et al. 2010, ApJ, 713, L164

Christiansen, J. L., Jenkins, J. M., Caldwell, D. A., et al. 2013, Kepler Data Characteristics Handbook, (KSCI - 19040 - 004), http: //archive. stsci . edu/kepler/documents.html

Cohen, O., Drake, J. J., Kashyap, V. L., Sokolov, I. V., \& Gombosi, T. I. 2010, ApJ, 723, L64

Coughlin, J. L., Thompson, S. E., Bryson, S. T., et al. 2014, AJ, 147, 119

Davies, G. R., Chaplin, W. J., Farr, W. M., et al. 2014, MNRAS, submitted

Désert, J.-M., Charbonneau, D., Demory, B.-O., et al. 2011, ApJS, 197, 14

Doğan, G., Metcalfe, T. S., Deheuvels, S., et al. 2013, ApJ, 763, 49

Dziembowski, W. 1977, Acta Astron., 27, 203

Epstein, C. R., \& Pinsonneault, M. H. 2014, ApJ, 780, 159

Esteves, L. J., De Mooij, E. J. W., \& Jayawardhana, R. 2013, ApJ, 772, 51

Fabrycky, D., \& Tremaine, S. 2007, ApJ, 669, 1298

Fabrycky, D. C., \& Winn, J. N. 2009, ApJ, 696, 1230

Faedi, F., Staley, T., Gómez Maqueo Chew, Y., et al. 2013, MNRAS, 433, 2097

Ferguson, J. W., Alexander, D. R., Allard, F., et al. 2005, ApJ, 623, 585

Ford, E. B., \& Rasio, F. A. 2008, ApJ, 686, 621

Foreman-Mackey, D., Hogg, D. W., Lang, D., \& Goodman, J. 2013, PASP, 125, 306

Formicola, A., Imbriani, G., Costantini, H., et al. 2004, Phys. Lett. B, 591, 61

Frandsen, S., Jones, A., Kjeldsen, H., et al. 1995, A\&A, 301, 123

Frasca, A., Fröhlich, H.-E., Bonanno, A., et al. 2011, A\&A, 532, A81

Freire Ferrero, R., Frasca, A., Marilli, E., \& Catalano, S. 2004, A\&A, 413, 657

Fröhlich, H.-E., Frasca, A., Catanzaro, G., et al. 2012, A\&A, 543, A146

Gandolfi, D., Collier Cameron, A., Endl, M., et al. 2012, A\&A, 543, L5 
M. N. Lund et al.: Asteroseismic inference on the spin-orbit misalignment and stellar parameters of HAT-P-7

Geweke, J. 1992, in Bayesian statistics, 4 (Oxford Univ. Press, New York), 169

Gilliland, R. L., Brown, T. M., Christensen-Dalsgaard, J., et al. 2010, PASP, 122, 131

Gizon, L., \& Solanki, S. K. 2003, ApJ, 589, 1009

Goldreich, P., Murray, N., \& Kumar, P. 1994, ApJ, 424, 466

Gough, D. O. 1977a, ApJ, 214, 196

Gough, D. O. 1977b, in Problems of Stellar Convection, eds. E. A. Spiegel, \&

J.-P. Zahn (Berlin: Springer Verlag), Lect. Notes Phys., 71, 57

Grec, G., Fossat, E., \& Pomerantz, M. A. 1983, Sol. Phys., 82, 55

Grevesse, N., \& Sauval, A. J. 1998, Space Sci. Rev., 85, 161

Gustafsson, B., Edvardsson, B., Eriksson, K., et al. 2008, A\&A, 486, 951

Hall, J. C. 2008, Liv. Rev. Sol. Phys., 5, 2

Handberg, R., \& Campante, T. L. 2011, A\&A, 527, A56

Handberg, R., \& Lund, M. N. 2014, MNRAS, accepted [arXiv: 1409. 1366]

Harvey, J. 1985, in Future Missions in Solar, Heliospheric \& Space Plasma Physics, eds. E. Rolfe, \& B. Battrick, ESA SP, 235, 199

Henden, A. A., Welch, D. L., Terrell, D., \& Levine, S. E. 2009, in AAS Meeting Abstracts, 214, 407.02

Hirano, T., Sanchis-Ojeda, R., Takeda, Y., et al. 2012, ApJ, 756, 66

Hirano, T., Sanchis-Ojeda, R., Takeda, Y., et al. 2014, ApJ, 783, 9

Høg, E., Fabricius, C., Makarov, V. V., et al. 2000, A\&A, 355, L27

Hou, F., Goodman, J., Hogg, D. W., Weare, J., \& Schwab, C. 2012, ApJ, 745, 198

Houdek, G. 2006, in Proc. SOHO 18/GONG 2006/HELAS I, Beyond the spherical Sun, ESA SP, 624,

Houdek, G. 2012, in Progress in Solar/Stellar Physics with Helio- and Asteroseismology, eds. H. Shibahashi, M. Takata, \& A. E. Lynas-Gray, ASP Conf. Ser., 462, 7

Houdek, G., \& Gough, D. O. 2002, MNRAS, 336, L65

Houdek, G., Balmforth, N. J., Christensen-Dalsgaard, J., \& Gough, D. O. 1999, A\&A, 351, 582

Huber, D., Carter, J. A., Barbieri, M., et al. 2013a, Science, 342, 331

Huber, D., Chaplin, W. J., Christensen-Dalsgaard, J., et al. 2013b, ApJ, 767, 127

Huber, D., Silva Aguirre, V., Matthews, J. M., et al. 2014, ApJS, 211, 2

Husnoo, N., Pont, F., Mazeh, T., et al. 2012, MNRAS, 422, 3151

Hut, P. 1981, A\&A, 99, 126

Iglesias, C. A., \& Rogers, F. J. 1996, ApJ, 464, 943

Karoff, C. 2008, Ph.D. Thesis, University of Aarhus, Denmark

Kippenhahn, R., Weigert, A., \& Weiss, A. 2013, Stellar Structure and Evolution

Kjeldsen, H. 1992, Ph.D. Thesis, University of Aarhus, Denmark

Kjeldsen, H., \& Frandsen, S. 1992, PASP, 104, 413

Kjeldsen, H., Bedding, T. R., \& Christensen-Dalsgaard, J. 2008, ApJ, 683, L175

Knaack, R., Fligge, M., Solanki, S. K., \& Unruh, Y. C. 2001, A\&A, 376, 1080

Koch, D. G., Borucki, W. J., Basri, G., et al. 2010, ApJ, 713, L79

Kraft, R. P. 1967, ApJ, 150, 551

Kunz, R., Fey, M., Jaeger, M., et al. 2002, ApJ, 567, 643

Lai, D., Foucart, F., \& Lin, D. N. C. 2011, MNRAS, 412, 2790

Ledoux, P. 1951, ApJ, 114, 373

Lund, M. N., Kjeldsen, H., Christensen-Dalsgaard, J., Handberg, R., \& Silva Aguirre, V. 2014, ApJ, 782, 2

Lundkvist, M., Kjeldsen, H., \& Silva Aguirre, V. 2014, A\&A, 566, A82

Magic, Z., Serenelli, A., Weiss, A., \& Chaboyer, B. 2010, ApJ, 718, 1378

Mardling, R. A. 2011, in IAU Symp. 276, eds. A. Sozzetti, M. G. Lattanzi, \& A. P. Boss, 238

Mathur, S., García, R. A., Morgenthaler, A., et al. 2013, A\&A, 550, A32

Mathur, S., García, R. A., Ballot, J., et al. 2014, A\&A, 562, A124

Matsumura, S., Peale, S. J., \& Rasio, F. A. 2010, ApJ, 725, 1995

McLaughlin, D. B. 1924, ApJ, 60, 22

McQuillan, A., Aigrain, S., \& Mazeh, T. 2013, MNRAS, 432, 1203

Meibom, S., Mathieu, R. D., \& Stassun, K. G. 2009, ApJ, 695, 679
Meibom, S., Mathieu, R. D., Stassun, K. G., Liebesny, P., \& Saar, S. H. 2011, ApJ, 733, 115

Miller, N., Fortney, J. J., \& Jackson, B. 2009, ApJ, 702, 1413

Morris, B. M., Mandell, A. M., \& Deming, D. 2013, ApJ, 764, L22

Morton, T. D., \& Johnson, J. A. 2011, ApJ, 729, 138

Nagasawa, M., Ida, S., \& Bessho, T. 2008, ApJ, 678, 498

Narita, N., Sato, B., Hirano, T., \& Tamura, M. 2009, PASJ, 61, L35

Narita, N., Kudo, T., Bergfors, C., et al. 2010, PASJ, 62, 779

Narita, N., Takahashi, Y. H., Kuzuhara, M., et al. 2012, PASJ, 64, L7

Nielsen, M. B., Gizon, L., Schunker, H., \& Karoff, C. 2013, A\&A, 557, L10

Noguchi, K., Aoki, W., Kawanomoto, S., et al. 2002, PASJ, 54, 855

Pál, A., Bakos, G. Á., Torres, G., et al. 2008, ApJ, 680, 1450

Patil, A., Huard, D., \& Fonnesbeck, C. J. 2010, Journal of Statistical Software, 35,1

Paxton, B., Bildsten, L., Dotter, A., et al. 2011, ApJS, 192, 3

Paxton, B., Cantiello, M., Arras, P., et al. 2013, ApJS, 208, 4

Ramírez, I., Michel, R., Sefako, R., et al. 2012, ApJ, 752, 5

Rogers, F. J., \& Nayfonov, A. 2002, ApJ, 576, 1064

Rogers, F. J., Swenson, F. J., \& Iglesias, C. A. 1996, ApJ, 456, 902

Rogers, T. M., Lin, D. N. C., \& Lau, H. H. B. 2012, ApJ, 758, L6

Rossiter, R. A. 1924, ApJ, 60, 15

Roxburgh, I. W., \& Vorontsov, S. V. 2003, A\&A, 411, 215

Salpeter, E. E. 1955, ApJ, 121, 161

Sanchis-Ojeda, R., Winn, J. N., Holman, M. J., et al. 2011, ApJ, 733, 127

Sanchis-Ojeda, R., Winn, J. N., Marcy, G. W., et al. 2013, ApJ, 775, 54

Schlaufman, K. C. 2010, ApJ, 719, 602

Shkolnik, E., Walker, G. A. H., Bohlender, D. A., Gu, P.-G., \& Kürster, M. 2005, ApJ, 622, 1075

Silva Aguirre, V., Ballot, J., Serenelli, A. M., \& Weiss, A. 2011a, A\&A, 529, A63

Silva Aguirre, V., Chaplin, W. J., Ballot, J., et al. 2011b, ApJ, 740, L2

Silva Aguirre, V., Casagrande, L., Basu, S., et al. 2012, ApJ, 757, 99

Silva Aguirre, V., Basu, S., Brandão, I. M., et al. 2013, ApJ, 769, 141

Simon, T., \& Landsman, W. 1991, ApJ, 380, 200

Skumanich, A. 1972, ApJ, 171, 565

Spiegelhalter, D. J., Best, N. G., Carlin, B. P., \& van der Linde, A. 2002, J. R. Stat. Soc. Ser. B Stat. Methodol., 64, 583

Steigman, G. 2010 [arXiv: 1008 . 4765]

Szabó, G. M., Szabó, R., Benkő, J. M., et al. 2011, ApJ, 736, L4

Torrence, C., \& Compo, G. P. 1998, Bull. Am. Meteorol. Soc., 79, 61

Torres, G., Fischer, D. A., Sozzetti, A., et al. 2012, ApJ, 757, 161

Toutain, T., \& Appourchaux, T. 1994, A\&A, 289, 649

Triaud, A. H. M. J., Collier Cameron, A., Queloz, D., et al. 2010, A\&A, 524, A 25

Valsecchi, F., \& Rasio, F. A. 2014, ApJ, 786, 102

Van Eylen, V., Kjeldsen, H., Christensen-Dalsgaard, J., \& Aerts, C. 2012, Astron. Nachr., 333, 1088

Van Eylen, V., Lindholm Nielsen, M., Hinrup, B., Tingley, B., \& Kjeldsen, H. 2013, ApJ, 774, L19

Van Eylen, V., Lund, M. N., Silva Aguirre, V., et al. 2014, ApJ, 782, 14

van Saders, J. L., \& Pinsonneault, M. H. 2013, ApJ, 776, 67

Vogt, S. S., Allen, S. L., Bigelow, B. C., et al. 1994, in Instrumentation in Astronomy VIII, eds. D. L. Crawford \& E. R. Craine, SPIE Conf. Ser., 2198, 362

Weber, E. J., \& Davis, Jr., L. 1967, ApJ, 148, 217

Weiss, A., \& Schlattl, H. 2008, Ap\&SS, 316, 99

Winn, J. N. 2010 [arXiv: 1001.2010 ]

Winn, J. N., Noyes, R. W., Holman, M. J., et al. 2005, ApJ, 631, 1215

Winn, J. N., Johnson, J. A., Albrecht, S., et al. 2009, ApJ, 703, L99

Winn, J. N., Fabrycky, D., Albrecht, S., \& Johnson, J. A. 2010, ApJ, 718, L145

Wright, J. T., Marcy, G. W., Butler, R. P., \& Vogt, S. S. 2004, ApJS, 152, 261 


\section{Appendix A: Power-spectrum modelling and optimisation}

\section{A.1. Modelling the power spectrum}

To describe the observed power spectral density of a mode peak in the frequency power spectrum, we use a standard Lorentzian function (see, e.g., Anderson et al. 1990; Gizon \& Solanki 2003) given by

$L_{n l m}(v)=H_{n l m}\left[1+\left(\frac{\left(v-v_{n l m}\right)}{\Gamma_{n l} / 2}\right)^{2}\right]^{-1}$.

The use of a Lorentzian function for the mode line profile comes from the nature of solar-like $p$-modes; the modes are stochastically driven by turbulent convection in the outer envelope after which they are intrinsically damped (see, e.g., Goldreich et al. 1994). In this equation, $H_{n l m}$ is the mode height, $v_{n l m}$ is the resonance frequency of the mode, while $\Gamma_{n l}$ is a measure of the damping rate of the mode and gives the FWHM of $L_{n l m}(v)$.

For slow stellar rotation the star is generally assumed to rotate as a rigid body and the modes will to first order be split as (Ledoux 1951)

$v_{n l m}=v_{n l}+m \frac{\Omega}{2 \pi}\left(1-C_{n l}\right) \approx v_{n l}+m v_{\mathrm{s}}$.

Here $m$ is the azimuthal order of the mode, $\Omega$ is the angular rotation rate of the star, and $C_{n l}$ is a dimensionless constant that describes the effect of the Coriolis force (the Ledoux constant). For high-order low-degree solar-like oscillations, like the ones we wish to analyse, this quantity is of the order $C_{n l}<10^{-2}$, and is therefore neglected. In this way we see that the splitting due to rotation between adjacent components of a multiplet will to a good approximation be given by $v_{\mathrm{s}}=\Omega / 2 \pi$.

In assuming equipartition of power between the components of a multiplet (i.e. no assumed preference in the excitation for prograde over retrograde propagating modes), it is possible to calculate the geometrical modulation of the relative visibility between the $2 l+1$ multiplet components as a function of $i_{\star}$ as (see, e.g., Dziembowski 1977; Gizon \& Solanki 2003)

$\mathcal{E}_{l m}\left(i_{\star}\right)=\frac{(l-|m|) !}{(l+|m|) !}\left[P_{l}^{|m|}\left(\cos i_{\star}\right)\right]^{2}$,

where $P_{l}^{m}(x)$ are the associated Legendre functions.

With this, the limit spectrum (noise-free) to be fit to the power spectrum is as expressed in Eq. (1) (see Fig. 2). By comparing Eqs. (A.1) and (1), we see that the height is given by $\mathcal{E}_{l m}(i) S_{n l}$. By assuming equipartition of energy between different radial orders, this can be written as

$H_{n l m}=\mathcal{E}_{l m}\left(i_{\star}\right) S_{n l}=\mathcal{E}_{l m}\left(i_{\star}\right) \tilde{V}_{l}^{2} \alpha_{l=0}(v)$.

The factor $\tilde{V}_{l}^{2}$ is a measure of the relative visibility in power (primarily set by partial cancellation) between non-radial and radial $(l=0)$ modes, while $\alpha_{l=0}(v)$ represents a (mainly) frequencydependent height modulation for the radial modes, generally represented by a Gaussian centred on the frequency of maximum oscillation power, $v_{\max }$.

\section{A.2. Optimisation procedure}

The fitting of Eq. (1) to the power spectrum is made in a Bayesian manner by mapping the posterior probability:

$p(\boldsymbol{\Theta} \mid D, I) \propto p(\boldsymbol{\Theta} \mid I) p(D \mid \Theta, I)$.
Here $p(\Theta \mid I)$ is the prior probability assigned to the parameters $\boldsymbol{\Theta}$ from any prior information $I$, and $p(D \mid \Theta, I)$ is the likelihood of the observed data $D$ given the parameters $\boldsymbol{\Theta}$. The posterior is approximated using the affine invariant MCMC sampler emcee (Foreman-Mackey et al. 2013; see also Hou et al. 2012). Using the emcee routine, the posterior distribution is mapped, after which parameter estimates are evaluated as the median of the respective marginalised distributions (see Sect. 4). In the sampling we enable the parallel tempering scheme of emcee and use five temperatures with tempering parameters set as $\beta_{i}=1.2^{1-i}$ (Benomar et al. 2009; Handberg \& Campante 2011). The affine invariant character of the emcee sampler ensures that it works efficiently in spite of linear parameter correlations, which are a problem for many MCMC algorithms. In our optimisation we make both a fit to a large (full fit) and a small (small fit) frequency range (see Sect. 4 for details). We employ 1500 (full fit) and 2000 (small fit) walkers, all initiated from a sampling of the prior distributions. Each walker is stopped after 10000 steps, after which we thin the chains by a factor of 10 (full fit) or 5 (small fit). We cut away a burn-in part of each chain based on the Geweke $^{7}$ statistics (Geweke 1992), and check for good mixing using the autocorrelation time of the chains and by performing a visual inspection of the traces of walkers in parameter space. We refer to Handberg \& Campante (2011) and references therein for further details on the MCMC nomenclature and ForemanMackey et al. (2013) for the specifics of the emcee sampler. To ensure better numerical stability we map the logarithm of the posterior with the description of the log-likelihood function from Anderson et al. (1990) and Toutain \& Appourchaux (1994). With regard to priors, we use top-hat priors for location parameters (e.g., $v_{n l}$ ) and scale invariant modified Jeffryes' priors for scale parameters (e.g., $S_{n 0}$ ). To decrease the computation time the limit-spectrum (Eq. (1)) was only fit to the frequency range that includes the identified oscillation modes (see Sect. 4.1 for further details). To better constrain the stellar noise-background in the relatively narrow range occupied by the oscillation modes, Eq. (2) was first fit to the power spectrum in the frequency range from $100-8496 \mu \mathrm{Hz}$ (the upper limit is the approximate Nyquist frequency of SC data) and included either one or two characteristic time-scales corresponding to the contributions from granulation only or granulation and faculae (this lower-limit frequency ensures that the activity component can be omitted). We also added a Gaussian function to Eq. (2) to account for the power excess from solar-like oscillations seen in HAT-P-7. Using the deviance information criterion (DIC; Spiegelhalter et al. 2002), we found that only one component is needed to describe of the background. The medians of the posteriors from this fit were then used to fix the background in the fit of Eq. (1).

\section{Appendix B: Line widths and visibilities}

\section{B.1. Line widths}

The line widths can have a strong impact on the estimated splitting because a small splitting could be equally well fit by a slightly larger line width. This is especially a problem when the splitting is smaller than the line width. We show in Fig. B.1 our fitted line widths for the radial $(l=0)$ modes and their associated uncertainties. As a sanity check, we may first compare the mode line width at the frequency of maximum power, $v_{\max }$, with the estimate from Eq. (2) of Appourchaux et al. (2012) and using combined values from their Table 2. Using the spectroscopic

Using the geweke module of PyMC (Patil et al. 2010). 


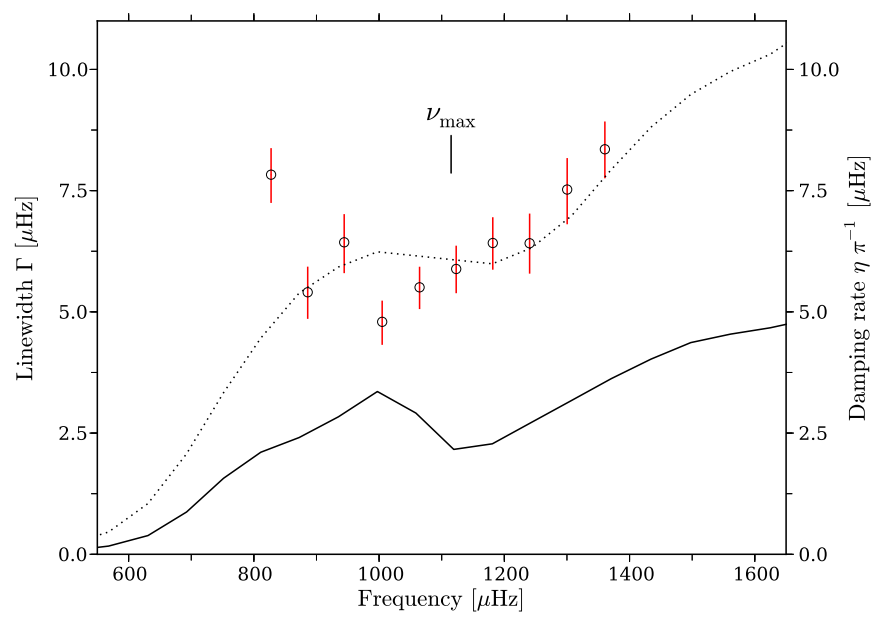

Fig. B.1. Measured line widths for radial-order modes are given by the open symbols as a function of frequency (left axis), and plotted with associated errors. Theoretical linear damping rates, multiplied by two, are shown by the solid curve (right axis). The dotted curve shows smoothed damping rates, multiplied by a factor of 2.2. Inputs to the theoretical model were taken from the best-fit GARSTEC model, e.g. the radius at the base of the surface convection zone $\left(R_{\mathrm{bcz}} / R_{\star}=0.862\right)$.

temperature of $T_{\mathrm{eff}}=6350 \pm 126 \mathrm{~K}$, yields $\Gamma \approx 3.5 \pm 1.1 \mu \mathrm{Hz}$. From the three $l=0$ modes closest to $v_{\max }$ we find (central) values of $\Gamma$ between 5.5 and $6.4 \mu \mathrm{Hz}$ (see Fig. B.1), which is higher than expected from the Appourchaux et al. (2012) formulae.

Additionally, we estimated linear damping rates, $\eta$, which we assumed to be approximately equal to half of the observed line widths, i.e. $\Gamma \simeq \eta \pi^{-1}$, if $\eta$ is in units of angular frequency. The outcome is shown in Fig. B.1. The computations included a full non-adiabatic treatment of the pulsations and convection dynamics. Both the convective heat and momentum (turbulent pressure) fluxes were treated consistently in the equilibrium and pulsation computations using the nonlocal generalisation of the timedependent convection model by Gough (1977a,b). The computations were carried out as described by Houdek et al. (1999) and Houdek \& Gough (2002). For the non-local convection parameters we adopted the values $a^{2}=900$ and $b^{2}=2000$, and the mixing-length paramter was calibrated to obtain the same depth of the surface convection zone as in the best GARSTEC model. For the anisotropy parameter, $\Phi$ (see Houdek \& Gough 2002), the value 2.50 was adopted.

After applying a median smoothing filter to $\eta \pi^{-1}$, with a width in frequency corresponding to five radial modes, the result of $2.8 \mu \mathrm{Hz}$ at $v_{\max }$ of the oscillation heights lies within the error bars of the observational scaling relation by Appourchaux et al. (2012). To fit the observations in Fig. B.1 we multiplied the median-smoothed estimates by a factor of 2.2 (dotted curve in Fig. B.1), which agrees with previous comparisons between line width observations and model estimates for hotter solar-like stars (see, e.g., Houdek 2006, 2012).

\section{B.2. Visibilities}

For the visibilities (see Appendix A), we estimate $\tilde{V}_{1}^{2}=1.39 \pm$ 0.08 and $\tilde{V}_{2}^{2}=0.46 \pm 0.07$ from the small fit. These agree reasonably well with the theoretical values of $\tilde{V}_{1}^{2} \approx 1.51$ and $\tilde{V}_{2}^{2} \approx 0.53$ estimated from the tables of Ballot et al. (2011). We do note, however, that this agreement is no guarantee for correct values because some stars deviate from the simple theoretical estimates (see, e.g., Lund et al. 2014). If we calculate the visibilities using the method described in Ballot et al. (2011), but adopt a quadratic limb-darkening (LD) law and measured LD-parameters from fits to the planetary transit by Van Eylen et al. (2013) and Morris et al. (2013), we obtain values that are slightly lower than those from theoretical LD parameters: $\tilde{V}_{1}^{2} \approx 1.46 \pm 0.02$ and $\tilde{V}_{2}^{2} \approx 0.46 \pm 0.01$. These values agree within the errors with the fit values, which is encouraging given the very simplified assumptions adopted in the Ballot et al. (2011) calculation, where for instance all non-adiabatic effects are neglected.

\section{Appendix C: Determining $(B-V)_{0}$}

We determined the photometric stellar parameters for HAT-P-7 by combining asteroseismic results with the Infrared flux method (IRFM) (see Silva Aguirre et al. 2011b, 2012). We adopted our seismic $\log g$ and the spectroscopic metallicity from Huber et al. (2013b, 2014) and used the IRFM implementation described in Casagrande et al. (2014), where different three-dimensional reddening maps are used to constrain extinction. At a distance of 320 pc (approximate distance to HAT-P-7 determined by Pál et al. 2008), reddening varies between $0.02<E(B-V)<0.03$.

Unfortunately, optical measurements of HAT-P-7 are quite uncertain, and depending on whether the Tycho2 (Høg et al. 2000) or APASS (Henden et al. 2009) photometry is used, the resulting $T_{\text {eff }}$ will vary anywhere between 6350 and $6650 \mathrm{~K}$. At the magnitude of our star, Tycho 2 photometry becomes increasingly uncertain (Høg et al. 2000) (although its $T_{\text {eff }}$ would be in overall good agreement with the spectroscopic estimate of $\left.T_{\text {eff }}=6350 \pm 126 \mathrm{~K}\right)$, while the APASS $(B-V)_{0}$ index (i.e., after correcting it for reddening) is almost as red as the solar one (Ramírez et al. 2012), thus suggesting a $T_{\text {eff }}$ close to solar. The higher $T_{\text {eff }}$ is, however, confirmed by the (reddening corrected) $J-K_{s}$ index of HAT-P-7 (indeed bluer than the solar one; Casagrande et al. 2012). From these considerations we thus discard the APASS $(B-V)_{0}$ as faulty, and adopt a photometric $T_{\text {eff }}=6500 \pm 150 \mathrm{~K}$, where the generous errors account for the discussed uncertainties. With this $T_{\text {eff }}$ and $[\mathrm{Fe} / \mathrm{H}]=0.26$, we can invert the colour- $T_{\text {eff }}-[\mathrm{Fe} / \mathrm{H}]$ relation of Casagrande et al. (2010), which returns an intrinsic (i.e., unreddened) colour of $(B-V)_{0}=0.455 \pm 0.040 \mathrm{mag}$. For spectroscopic $T_{\text {eff }}$ and uncertainty, its $(B-V)_{0}=0.495 \pm 0.022$.

Synthetic photometry offers an alternative way of assessing the $(B-V)_{0}$ colour for HAT-P-7 (all synthetic quantities are obtained by interpolating at the "known" physical parameters of the star, and are thus unaffected by reddening). We use the large grid of MARCS (Gustafsson et al. 2008) synthetic colours and interpolation routines provided by Casagrande \& VandenBerg (2014) to infer the $(B-V)_{0}$ index of HAT-P-7, for the spectroscopic parameters and asteroseismic $\log g$.

To estimate the uncertainty in the synthetic $(B-V)_{0}$, we also compute all possible $T_{\text {eff }}$ and $[\mathrm{Fe} / \mathrm{H}]$ combinations allowed by the spectroscopic uncertainties (while the seismic $\log g$ is so precisely known that changing it makes no difference). With this procedure, we obtain $(B-V)_{0}=0.498 \pm 0.020$, in excellent agreement with the estimate from the empirical colour- $T_{\text {eff }}-[\mathrm{Fe} / \mathrm{H}] \mathrm{re}-$ lation when using the spectroscopic $T_{\text {eff }}$.

For the gyrochronology calculation we used the value $(B-V)_{0}=0.495 \pm 0.022$ from the IRFM when using the spectroscopic $T_{\text {eff. }}$. 


\section{Appendix D: Peak-bagging results}

Table D.1. Frequencies extracted from the MCMC peak-bagging.

\begin{tabular}{llll}
\hline \hline$n$ & $l=0[\mu \mathrm{Hz}]$ & $l=1[\mu \mathrm{Hz}]$ & $l=2[\mu \mathrm{Hz}]$ \\
\hline 9 & & $623.18_{-0.43}^{+0.35}$ & \\
10 & $651.42_{-0.48}^{+0.50}$ & $683.36_{-0.41}^{+0.38}$ & $710.83_{-0.55}^{+0.56}$ \\
11 & $715.37_{-0.34}^{+0.34}$ & $740.71_{-0.35}^{+0.35}$ & $767.85_{-0.95}^{+1.01}$ \\
12 & $771.46_{-0.87}^{+0.79}$ & $796.46_{-0.41}^{+0.42}$ & $824.85_{-0.91}^{+1.04}$ \\
13 & $827.89_{-0.51}^{+0.45}$ & $853.91_{-0.26}^{+0.27}$ & $882.37_{-0.61}^{+0.69}$ \\
14 & $886.13_{-0.29}^{+0.28}$ & $911.71_{-0.23}^{+0.23}$ & $940.68_{-0.59}^{+0.66}$ \\
15 & $944.26_{-0.43}^{+0.39}$ & $971.74_{-0.19}^{+0.18}$ & $1000.49_{-0.57}^{+0.55}$ \\
16 & $1005.00_{-0.25}^{+0.24}$ & $1031.51_{-0.16}^{+0.17}$ & $1059.36_{-0.54}^{+0.51}$ \\
17 & $1064.86_{-0.27}^{+0.25}$ & $1090.92_{-0.17}^{+0.17}$ & $1118.71_{-0.41}^{+0.41}$ \\
18 & $1123.36_{-0.27}^{+0.27}$ & $1149.77_{-0.18}^{+0.19}$ & $1177.55_{-0.75}^{+0.71}$ \\
19 & $1181.74_{-0.28}^{+0.29}$ & $1208.19_{-0.20}^{+0.20}$ & $1236.32_{-0.53}^{+0.58}$ \\
20 & $1240.64_{-0.31}^{+0.32}$ & $1267.67_{-0.25}^{+0.23}$ & $1297.08_{-0.73}^{+0.89}$ \\
21 & $1300.45_{-0.53}^{+0.47}$ & $1327.44_{-0.31}^{+0.30}$ & $1356.65_{-0.64}^{+0.74}$ \\
22 & $1360.68_{-0.53}^{+0.50}$ & $1388.21_{-0.37}^{+0.38}$ & $1416.94_{-0.96}^{+1.02}$ \\
23 & $1421.92_{-0.77}^{+0.76}$ & $1448.75_{-0.47}^{+0.50}$ & $1478.18_{-1.21}^{+1.21}$ \\
24 & $1482.68_{-0.92}^{+0.90}$ & $1510.15_{-0.58}^{+0.55}$ & $1539.50_{-1.15}^{+0.93}$ \\
25 & $1545.14_{-0.94}^{+0.84}$ & $1568.95_{-0.64}^{+0.64}$ & $1595.15_{-1.30}^{+0.77}$ \\
26 & $1602.95_{-1.23}^{+0.46}$ & & \\
\hline & & & \\
\hline
\end{tabular}

Notes. Note: From the échelle diagram in Fig. 3 it seems that the signal fitted as the lowest $l=0$ mode might originate from an $l=2$ mode. 